\title{
Continuous Reinhardt domains from a Jordan viewpoint
}

\author{
by \\ L. L. Stachó (Szeged)
}

\begin{abstract}
As a natural extension of bounded complete Reinhardt domains in $\mathbb{C}^{N}$ to spaces of continuous functions, continuous Reinhardt domains (CRD) are bounded open connected solid sets in commutative $C^{*}$-algebras with respect to the natural ordering. We give a complete parametric description for the structure of holomorphic isomorphisms between CRDs and characterize the partial Jordan triple structures which can be associated with some CRDs. On the basis of these results, we test two conjectures concerning the Jordan structure of bounded circular domains. It turns out that both the problems of bidualization and unique extension of inner derivations have positive solution in the setting of CRDs.
\end{abstract}

1. Introduction. A classical complete Reinhardt domain is an open connected subset in the space $\mathbb{C}^{n}$ of all complex $n$-tuples, which is invariant under all coordinate multiplications $\left(z_{1}, \ldots, z_{n}\right) \mapsto\left(\lambda_{1} z_{1}, \ldots, \lambda_{n} z_{n}\right)$ with $\left|\lambda_{1}\right|, \ldots,\left|\lambda_{n}\right| \leq 1$. If we regard $\mathbb{C}^{n}$ as the complex ordered space of functions $z:\{1, \ldots, n\} \rightarrow \mathbb{C}$, this property can be stated as

$$
f \in D \text { and }|g| \leq|f| \Rightarrow g \in D \text {. }
$$

Postulating (CR) for the order absolute value, we can speak of bounded complete Reinhardt domains in complex Banach lattices in a natural manner.

1974 Sunada [18] obtained a rather thorough description of classical bounded Reinhardt domains containing the origin from the viewpoint of holomorphic equivalence. Later on several authors investigated holomorphic equivalence of generalized Reinhardt domains in atomic Banach lattices $[1$, $2,12]$. Motivated by an interesting work of Vigué [19] on the possible lack of symmetry of continuous products of discs with different radii, in [17] we introduced the concept of continuous Reinhardt domains (CRD for short).

2000 Mathematics Subject Classification: Primary 32M12; Secondary 46G20, 32M15.

Key words and phrases: Jordan triple, holomorphic automorphism, commutative, $C^{*}$ algebra, continuous Reinhardt domain, bidual, derivation.

Supported by the Hungarian research grant No. OTKA T/17 48753. 
By definition, a CRD is a bounded complete Reinhardt domain in the $C^{*}$ algebra of all bounded continuous functions over some topological space, or more generally, in a commutative $C^{*}$-algebra. In [17] we have shown that a symmetric CRD is a continuous mixture of finite-dimensional Euclidean balls, essentially more involved than direct sums of topological products of balls. In [7] we found matrix representations for linear isomorphisms between two symmetric CRDs. To achieve these results we intensively used the Jordan theory of the bidual embedding of symmetric domains. However, the main points of both Sunada's and Vigué's papers concern the nonsymmetric case. Recently, based upon the Lie theory of Hermitian operators in the dual space, in [16] we managed to extend the matrix representations of [7] to a Banach-Stone type theorem on isomorphisms of general Banach lattice normed commutative $C^{*}$-algebras. This result includes implicitly the description of all possible linear isomorphisms between CRDs because the convex hull of any CRD can be regarded as the unit ball of some lattice norm in a commutative $C^{*}$-algebra and linear isomorphisms preserve convex hulls.

The aim of this paper is a description of all possible holomorphic equivalences of CRDs. According to a classical result of [4], every bounded circular domain and hence even a non-symmetric CRD admits a natural partial Jordan *-triple structure, a so-called partial $J B^{*}$-triple, which gives rise to the description of its complete holomorphic vector fields - a crucial piece of information about its holomorphic geometry. In particular, every holomorphic isomorphism between two bounded circular domains is the composition of a linear isomorphism with the exponential of a suitable complete holomorphic vector field over one of the two domains.

In Section 2 we review the basic material $[4,13,14]$ concerning partial $J B^{*}$-triples. The bidual embedding arguments used in [17] to treat the Jordan structure in the symmetric case are not available for general CRDs. Although Dineen [5] and Barton-Timoney [3] established a satisfactory bidual Jordan theory for all bounded convex circular domains already in 1986, it is still one of the fundamental open questions in geometric Jordan theory, with no interesting partial results so far, whether the canonical partial triple product associated with any non-convex bounded circular domain extends in a weak ${ }^{*}$-continuous manner to the canonical partial triple product of some bounded circular domain in the bidual. Instead, in Section 3 we develop an alternative approach for determining the partial $J B^{*}$-triple product associated with a CRD. The conclusion, Theorem 3.5, is an integral representation of this triple product. In classical finite-dimensional complex analysis, Reinhardt domains are popular test objects for conjectures. In the second half of the paper we use this integral representation as a starting point to solve the special case of two open problems on bounded circular domains in the setting of CRDs. 
The first problem we treat has its origin in a work of Panou [10] where it is shown that every inner derivation of the Jordan triple associated with the symmetric part of a finite-dimensional bounded circular domain admits a unique extension to an inner derivation of the partial Jordan triple associated with the whole domain. Though it is natural to expect that the analog holds in general Banach spaces, the only known infinite-dimensional results concern domains with nearly atomic symmetric part [15]. On the basis of Theorem 3.5 along with the fine structure description of the Jordan triple product associated with a symmetric CRD [7], in Section 4 we can establish immediately that the partial Jordan triple of a CRD has the unique extension property for inner derivations.

The second question we solve for CRDs is the above mentioned open problem of the Jordan structure of the second dual of a partial $J B^{*}$-triple. First, in Section 5 we refine Theorem 3.5 to a natural extension of the results for symmetric CRDs given in [7] whose proofs relied upon some bidual considerations in [17]. By proceeding the opposite way, in Section 6 we apply the fine structure description obtained in Section 5 along with function representations of $\mathcal{C}_{0}(\Omega)^{\prime \prime}$ spaces to establish that the Jordan triple product associated with a CRD admits a separately weak*-continuous bidual extension which can be regarded as the canonical Jordan triple of some not necessarily unique CRD.

In the course of Section 5 we apply a Riesz type representation theorem for positive multilinear functionals on products of $\mathcal{C}_{0}$-spaces which seems to have never been stated explicitly in the literature. Actually the result we need is contained implicitly in a recent work of Villanueva [20]. We close the paper with an Appendix including a short direct proof.

2. Preliminaries on partial $J B^{*}$-triples. Recall [14] that given a complex Banach space $E$ (with norm $\|\cdot\|$ ), the tuple $\left(E, E_{0},\{\ldots\}\right.$ ) is called a partial Jordan ${ }^{*}$-triple if $E_{0}$ is a closed complex subspace of $E$, and $\{\ldots\}$ is a continuous operation $E \times E_{0} \times E \rightarrow E$ with the following properties:

(J1) $\{x a y\}$ is symmetric bilinear in the variables $x, y(\in E)$, conjugate linear in $a\left(\in E_{0}\right)$ and $\left\{E_{0} E_{0} E_{0}\right\} \subset E_{0}$;

(J2) the Jordan identity holds, i.e. for all $a, b, c \in E_{0}$ and $x, y \in V$,

$$
\{a b\{x c y\}=\{\{a b x\} c y\}-\{x\{b a c\} y\}+\{x c\{a b z\}\}
$$

(J3) we have the weak associativity

$$
\{\{x a x\} b x\}=\{x a\{x b x\}\}, \quad a, b \in E_{0}, x \in E .
$$

Notice that in the case of full Jordan ${ }^{*}$-triples, i.e. if $E_{0}=E$, axiom (J3) is a consequence of (J2) (see e.g. [6, Ch. 10]). The geometric importance of partial Jordan *-triples relies upon the fact established first implicitly in 
$[4,9,6]$ that given any bounded circular domain $D$ in a Banach space $E$, there is a necessarily unique partial Jordan ${ }^{*}$-triple $\left(E, E_{D},\{\ldots\}_{D}\right)$ called the canonical partial Jordan *-triple of $D$ such that $D \cap E_{D}$ consists of the centers of holomorphic symmetries of $D$ and $\lim _{t \downarrow 0} S_{t a} S_{0}(x)=a-\{x a x\}_{D}$ for all $a \in E_{D}$ and $x \in E$ where $S_{c}$ denotes the holomorphic symmetry of $D$ with center $c \in D \cap E_{D}$. We say that $\left(E, E_{0},\{\ldots\}\right)$ is a partial $J B^{*}$-triple if it is a subtriple of some canonical partial Jordan ${ }^{*}$-triple $\left(E, E_{D},\{\ldots\}_{D}\right)$. In other words, this means that all the vector fields $[a-\{x a x\}] \partial / \partial x, a \in E_{0}$, are complete in a suitable bounded circular domain $D(\subset E)$. This terminology is in accordance with the customary use of the term $J B^{*}$-triple for full Jordan triples. Indeed, by Kaup's Riemann mapping theorem [8], in the case $E=E_{D}$ the domain $D$ is necessarily convex and hence the carrier space $E$ can be renormed so that $D$ becomes the unit ball and the usual $C^{*}$ and hermitian positivity axioms are satisfied. By the results of $[13,14]$, we have a complete axiomatic description of partial $J B^{*}$-triples. A partial Jordan *-triple $\left(E, E_{0},\{\ldots\}\right)$ is a partial $J B^{*}$-triple if and only if

(J4) the operators $L(a): x \mapsto\{a a x\}, a \in E_{0}$, have spectrum $\geq 0$ with

$$
\inf _{\|a\|=1}\|L(a) a\| \neq 0
$$

(J5) $L(a) \in \operatorname{Her}(B), a \in E_{0}$, for some bounded circular domain $B$.

It is well-known that the domain $B$ in (J5) can be chosen to be convex and such that its gauge function $\|\cdot\|_{B}$ should satisfy the $C^{*}$-axiom

$\left(\mathrm{J} 4^{\prime}\right) \operatorname{Sp}(L(a)) \geq 0$ with $\|L(a) a\|_{B}=\|a\|_{B}^{3}$ for all $a \in E_{0}$.

Given any bounded circular domain $B$ satisfying (J5), there exists $\varepsilon>0$ such that for any $\delta \in(0, \varepsilon),\left(E, E_{0},\{\ldots\}\right)$ is a subtriple of $\left(E, E_{D_{\delta}},\{\ldots\}_{D_{\delta}}\right)$ with the bounded circular domain

$$
D_{\delta}:=\bigcup_{a \in E_{0}}[\exp ((a-\{x a x\}) \partial / \partial x)](\delta B) .
$$

Our next aim will be to describe the canonical partial $J B^{*}$-triples of CRDs. Recall [4] that the group $\operatorname{Aut}\left(E, E_{0},\{\ldots\}\right):=\left\{L \in \mathcal{L}(E): L E_{0} \subset E_{0}\right.$, $L\{x a y\}=\{(L x)(L a)(L y)\}$ for $\left.a \in E_{0}, x, y \in E\right\}$ of all automorphisms of the triple $\mathbf{E}=\left(E, E_{0},\{\ldots\}\right)$ coincides with the set of all injective linear transformations $L: E \rightarrow E$ such that $L D=D$ whenever $\mathbf{E}$ is the canonical $J B^{*}$-triple of a bounded circular domain $D$. In particular, if $D \subset \mathcal{C}_{0}(\Omega)$ is a CRD, all multiplications with continuous functions of absolute value one belong to $\operatorname{Aut}\left(E, E_{D},\{\ldots\}_{D}\right)$. So first we consider the effect of linear automorphisms on the construction (2.1). 
2.2. Lemma. Let $\left(E, E_{0},\{\ldots\}\right)$ be a partial $J B^{*}$-triple and $\Psi$ a bounded subgroup of $\operatorname{Aut}\left(E, E_{0},\{\ldots\}\right)$. Then there exists a $\Psi$-invariant bounded circular domain $D \subset E$ such that $\left(E, E_{0},\{\ldots\}\right)$ is a subtriple of $\left(E, E_{D},\{\ldots\}_{D}\right)$.

Proof. Choose a bounded circular domain $B$ in $E$ satisfying axiom (J5). Define $B_{1}:=\bigcup_{\psi \in \Psi} \psi B$. Since $\Phi$ is a bounded group of linear mappings, $B_{1}$ is a bounded $\Psi$-invariant circular domain in $E$. Given any $a \in E_{0}$ and $\psi \in \Psi$, since $\psi \in \operatorname{Aut}\left(E, E_{0},\{\ldots\}\right)$, we have $L\left(\psi^{-1} a\right)=\psi^{-1} L(a) \psi$. Since $\psi^{-1} a \in E_{0}$, by axiom (J5) it follows that $\exp \left(i t L\left(\psi^{-1} a\right)\right) \psi B=\psi B, t \in \mathbb{R}$. Since $\psi^{-1} a$ can be any element in $E_{0}$, we also get $\exp (i t L(a)) \psi B=\psi B$ for all $a \in E_{0}, t \in \mathbb{R}$ and $\psi \in \Psi$. That is, $L(a) \in \operatorname{Her}(\psi B), \psi \in \Psi$, and hence $L(a) \in \operatorname{Her}\left(\bigcup_{\phi \in \Psi} \psi B\right)=\operatorname{Her}\left(B_{1}\right)$ for all $a \in E_{0}$. Thus the domain $B_{1}$ satisfies axiom (J5) and we can use it in the construction (2.1) instead of $B$ with some $\delta>0$. Given any $\psi \in \Psi$, it only remains to prove that

$$
\psi \bigcup_{a \in E_{0}}[\exp ((a-\{x a x\}) \partial / \partial x)]\left(\delta B_{1}\right)=\bigcup_{a \in E_{0}}[\exp ((a-\{x a x\}) \partial / \partial x)]\left(\delta B_{1}\right) .
$$

However, this is again a direct consequence of the facts $\psi\left(\delta B_{1}\right)=\delta B_{1}$ and

$$
\psi \exp ((a-\{x a x\}) \partial / \partial x)=[\exp ((\psi a-\{x(\psi a) x\}) \partial / \partial x)] \psi .
$$

The latter identity follows from the relation $\psi \in \operatorname{Aut}\left(E, E_{0},\{\ldots\}\right)$.

3. Integral formula for the canonical partial triple product for a CRD. Let $\left(E, E_{0},\{\ldots\}\right)$ denote a fixed partial $J B^{*}$-triple over $E:=$ $\mathcal{C}_{0}(\Omega)$ with a locally compact topological Hausdorff space $\Omega$. Throughout this section we assume the Reinhardt property

$$
\Psi \subset \operatorname{Aut}\left(E, E_{0},\{\ldots\}\right) \quad \text { where } \quad \Psi:=\{\psi \cdot: \psi \in \mathcal{C}(\Omega),|\psi|=1\}
$$

and $\psi$. denotes the multiplication operator $\mathcal{C}_{0}(\Omega) \ni f \mapsto \psi f$. As we mentioned, the canonical partial $J B^{*}$-triple $\left(E, E_{D},\{\ldots\}_{D}\right)$ of any CRD $D$ has property $(\mathrm{R})$. Moreover, from Lemma $2.2,\left(E, E_{0},\{\ldots\}\right)$ can be regarded as a subtriple of the canonical $J B^{*}$-triple of some CRD in $\mathcal{C}_{0}(\Omega)$.

As a first consequence of $(\mathrm{R})$, we have $e^{i t \phi} E_{0} \subset E_{0}$ and

$$
e^{i t \phi}\{x a y\}=\left\{\left(e^{i t \phi} x\right)\left(e^{i t \phi} a\right)\left(e^{i t \phi} y\right)\right\}, \quad t \in \mathbb{R},
$$

for any bounded continuous function $\phi: \Omega \rightarrow \mathbb{R}$ (with $a \in E_{0}, x, y \in E$ ). Hence derivation with respect to the variable $t$ yields

$$
\psi E_{0} \subset E_{0}, \quad \psi\{x a y\}=\{(\psi x) a y\}-\{x(\bar{\psi} a) y\}+\{x a(\psi y)\}
$$

for all bounded continuous functions $\psi: \Omega \rightarrow \mathbb{C}$. In particular $E_{0}$ is a closed ideal in $\mathcal{C}_{0}(\Omega)$ regarded as a commutative $C^{*}$-algebra with the pointwise product of functions. Therefore necessarily

$$
E_{0}=\mathcal{C}_{0}\left(\Omega_{0}\right):=\left\{f \in \mathcal{C}_{0}(\Omega): f\left(\Omega \backslash \Omega_{0}\right)=0\right\}
$$

with the open set $\Omega_{0}:=\left\{\omega \in \Omega: \exists a \in E_{0} a(\omega) \neq 0\right\}$. 
3.2. Lemma. $\{x a y\}(\omega)=0$ whenever $x(\omega)=y(\omega)=0$.

Proof. By the symmetry (J1), it suffices to prove the statement for $x=y$. Furthermore, by the continuity of the triple product and since continuous functions vanishing at $\omega(\in \Omega)$ can be uniformly approximated by continuous functions vanishing on some neighborhood of $\omega$, it suffices to see that $\{x a x\}(\omega)=0$ if $x(U)=0$ for some neighborhood $U \subset \Omega$ of $\omega$.

Assume $\omega \in U$ where $U$ is open in $\Omega, x \in E$, and $x(U)=0$. Choose a compact neighborhood $V$ of $\omega$ within $U$ and let $\phi: \Omega \rightarrow[0,1]$ be a continuous function such that $\phi(\omega)=1$ and $\phi(\Omega \backslash V)=0$. Observe that if $c \in E_{0}$ is a function with $c(V)=0$ then $\phi x=\phi c=0$ and, by (5.1),

$$
\{x c x\}(\omega)=\phi\{x c x\}(\omega)=2\{(\phi x) c x\}(\omega)-\{x(\phi c) x\}(\omega)=0 .
$$

Consider any $a \in E_{0}$. Choose a continuous function $\psi: \Omega \rightarrow[0,1]$ with $\psi(V)=0$ and $\psi(\Omega \backslash U)=1$. Since $\psi x=x$, applying the function $c:=\psi a$ vanishing on $V$ we get

$$
\begin{aligned}
0 & =\psi\{x a x\}(\omega)=2\{(\psi x) a x\}(\omega)-\{x(\psi a) x\}(\omega) \\
& =2\{x a x\}-\{x c x\}=2\{x a x\} .
\end{aligned}
$$

3.3. Corollary. We have

$$
\{x a y\}=\frac{1}{2} x\{z(\bar{y} a) z\}+\frac{1}{2} y\{z(\bar{x} a) z\} \quad \text { if } z \in E \text { with } x z=x \text { and } y z=y \text {. }
$$

Proof. Suppose $x z=x$ and $y z=y$. For any $\omega \in \Omega$ apply Lemma 3.2 to the functions $x_{\omega}:=x-x(\omega) z$ and $y_{\omega}:=y-y(\omega) z$ satisfying $x_{\omega}(\omega)=$ $y_{\omega}(\omega)=0$ to get

$$
\begin{aligned}
0 & =\left\{x_{\omega} a y_{\omega}\right\}(\omega)=\{[x-x(\omega) z] a[y-y(\omega) z]\}(\omega) \\
& =\{x a y\}(\omega)+x(\omega) y(\omega)\{z a z\}(\omega)-x(\omega)\{z a y\}(\omega)-y(\omega)\{z a x\}(\omega) .
\end{aligned}
$$

Thus, everywhere on $\Omega$,

$$
\{x a y\}=-x y\{z a z\}+x\{z a y\}+y\{z a x\} .
$$

Observe that, by (3.1) and since $y z=y$, here we have

$$
x y\{z a z\}=x[2\{y a z\}-\{z(\bar{y} a) z\}]
$$

and similarly $y x\{z a z\}=y[2\{x a z\}-\{z(\bar{x} a) z\}]$. Therefore

$$
\begin{aligned}
x y\{z a z\} & =x\{z a y\}+y\{z a x\}-\frac{1}{2}\{z(\bar{x} a) z\}-\frac{1}{2}\{z(\bar{y} a) z\}, \\
\{x a y\} & =-x y\{z a z\}+x\{z a y\}+y\{z a x\}=\frac{1}{2} x\{z(\bar{y} a) z\}+\frac{1}{2} y\{z(\bar{x} a) z\} .
\end{aligned}
$$

3.4. Lemma. The triple product $\{\ldots\}$ is positive in the sense that

$$
x, a, y \geq 0 \Rightarrow\{x a y\} \geq 0 .
$$


Proof. Fix $0 \leq x, y \in E$ and $0 \leq a \in E_{0}$. Since the functions with compact support are dense in $\mathcal{C}_{0}$-spaces, we may assume

$$
\operatorname{supp}(x), \operatorname{supp}(y) \operatorname{compact} \subset \Omega, \quad \operatorname{supp}(a) \text { compact } \subset \Omega_{0} .
$$

Then we can choose $0 \leq x_{0}, x_{1}, y_{0}, y_{1}, z \in \mathcal{C}_{0}(\Omega)$ with compact support such that

$$
\begin{aligned}
& x=x_{0}+x_{1}, \quad y=y_{0}+y_{1}, \\
& \operatorname{supp}\left(x_{0}\right), \operatorname{supp}\left(y_{0}\right) \subset \Omega_{0}, \\
& \operatorname{supp}\left(x_{1}\right) \cap \operatorname{supp}(a)=\operatorname{supp}\left(y_{1}\right) \cap \operatorname{supp}(a)=\emptyset, \\
& \operatorname{supp}(x) \cup \operatorname{supp}(y) \cup \operatorname{supp}(a) \subset\{\zeta \in \Omega: z(\zeta)=1\} .
\end{aligned}
$$

By (J1) we have $\{x a y\}=\sum_{k, l=0}^{1}\left\{x_{k} a y_{l}\right\}$. Here $\left\{x_{0} a y_{0}\right\} \geq 0$ for the following reasons. The subtriple $\left(E_{0}, E_{0},\{\ldots\} \mid E_{0}^{3}\right)$ is a $J B^{*}$-triple with $\Psi \subset$ $\operatorname{Aut}\left(E_{0}, E_{0},\{\ldots\} \mid E_{0}^{3}\right)$. Therefore it is necessarily the canonical $J B^{*}$-triple of a bounded symmetric continuous Reinhardt domain in $E_{0}=\mathcal{C}_{0}\left(\Omega_{0}\right)$. However, by [17, Theorem 2] the triple product is non-negative for nonnegative functions for symmetric CRDs. Thus indeed $\left\{x_{0} a y_{0}\right\} \geq 0$ since $a, x_{0}, y_{0} \in E_{0}$. On the other hand, by Corollary 3.3,

$$
\left\{x_{1} a y_{1}\right\}=\frac{1}{2} x_{1}\left\{z\left(\bar{y}_{1} a\right) z\right\}+\frac{1}{2} y_{1}\left\{z\left(\bar{x}_{1} a\right) z\right\}=0
$$

because $\bar{x}_{1} a=\bar{y}_{1} a=0$. It only remains to see that $\left\{x_{0} a y_{1}\right\} \geq 0$ (since the proof of $\left\{x_{1} a y_{0}\right\}=\left\{y_{0} a x_{1}\right\} \geq 0$ is analogous). Define

$$
c:=\sqrt{x_{0} a} \text {. }
$$

Since $\operatorname{supp}(c) \subset \operatorname{supp}(a) \subset \Omega_{0}$, we have $c \in E_{0}$ and $c z=c$. By Corollary 3.3 (applied with $y_{1}$ instead of $y$ and first with $c$ instead both of $a$ and $x$ and then with $x_{0}$ instead of $x$ ),

$$
\begin{gathered}
\left\{c c y_{1}\right\}=\frac{1}{2} c\left\{z\left(y_{1} c\right) z\right\}+\frac{1}{2} y_{1}\left\{z\left(c^{2}\right) z\right\}=\frac{1}{2} y_{1}\left\{z\left(c^{2}\right) z\right\}, \\
\left\{x_{0} a y_{1}\right\}=\frac{1}{2} x_{0}\left\{z\left(y_{1} a\right) z\right\}+\frac{1}{2} y_{1}\left\{z\left(x_{0} a\right) z\right\}=\frac{1}{2} y_{1}\left\{z\left(x_{0} a\right) z\right\}
\end{gathered}
$$

because $y_{1} a=y_{1} c=0$. That is,

$$
\left\{x_{0} a y_{1}\right\}=\left\{c c y_{1}\right\}=\frac{1}{2} y_{1}\left\{z\left(x_{0} a\right) z\right\} .
$$

According to $(\mathrm{J} 4), \operatorname{Sp}(L(c)) \geq 0$. However, it is a basic fact about the spectra of multipliers in commutative Banach algebras that

$$
u(X) \subset \operatorname{Sp}\left[\mathcal{C}_{0}(X) \ni f \mapsto u f\right] \quad \text { if } X \text { open } \subset \Omega \text { and } u \in \mathcal{C}_{0}(\Omega) .
$$

In particular, by taking $X:=\Omega \backslash \operatorname{supp}(a)$ and $u:=\left\{z\left(x_{0} a\right) z\right\}$ we have

$$
\left\{z\left(x_{0} a\right) z\right\}(\Omega \backslash \operatorname{supp}(a)) \subset \operatorname{Sp} L(c) \subset[0, \infty) .
$$

Thus $\left\{z\left(x_{0} a\right) z\right\} \geq 0$ on $\operatorname{supp}\left(y_{1}\right)$ and hence $2\left\{x_{0} a y_{1}\right\}=y_{1}\left\{z\left(x_{0} a\right) z\right\} \geq 0$.

We can summarize the results of this section in the following theorem. 
3.5. Theorem. Let $\Omega$ be a locally compact space, $E:=\mathcal{C}_{0}(\Omega)$ and suppose $\left(E, E_{0},\{\ldots\}\right)$ is a partial $J B^{*}$-triple with the Reinhardt property $(\mathrm{R})$. Then there exists an open subset $\Omega_{0}$ in $\Omega$ such that $E_{0}=\{f \in E$ : $\left.f\left(\Omega \backslash \Omega_{0}\right)=0\right\}$. Given any $\omega \in \Omega$, there is a (unique) positive Radon measure $\mu_{\omega}$ on $\Omega_{0}$ with total mass $\leq M:=\sup _{0 \leq x, a, y \leq 1} \max \{x a y\}$ and

$$
\{x a y\}(\omega)=\frac{1}{2} x(\omega) \int \bar{a} y d \mu_{\omega}+\frac{1}{2} y(\omega) \int \bar{a} x d \mu_{\omega}, \quad x, y \in E, a \in E_{0} .
$$

Proof. We have already established the relation $E_{0}=\mathcal{C}_{0}\left(\Omega_{0}\right)$ and the positivity of the triple product in the sense of Lemma 3.4. Fix $\omega \in \Omega$. According to $[20]\left({ }^{1}\right)$, the positivity of the bounded 3-linear functional $(x, a, y) \mapsto\{x \bar{a} y\}(\omega)$ implies the existence of a positive Radon measure $\nu_{\omega}$ of finite total variation on $\Omega \times \Omega_{0} \times \Omega$ such that

$$
\{x \bar{a} y\}(\omega)=\int x \otimes a \otimes y d \nu_{\omega}, \quad x, y \in E, a \in E_{0},
$$

where $x \otimes a \otimes y$ denotes the function $(\xi, \alpha, \eta) \mapsto x(\xi) a(\alpha) y(\eta)$ on $\Omega \times \Omega_{0} \times \Omega$. It is well-known that $\nu_{\omega}\left(\Omega \times \Omega_{0} \times \Omega\right)=\sup \left\{\int x \otimes a \otimes y d \nu_{\omega}: a \in \mathcal{C}_{0}\left(\Omega_{0}\right), x, y \in\right.$ $\left.\mathcal{C}_{0}(\Omega), 0 \leq a, x \leq 1\right\}=M$. By the inner compact regularity of Radon measures, given any functions $x, y \in E$ with compact support and $a \in E_{0}$, we can choose an increasing sequence $K_{1} \subset K_{2} \subset \cdots \subset \Omega$ of compact sets such that $\operatorname{supp}(x) \cup \operatorname{supp}(y) \subset K_{1}$ and $\lim _{n \rightarrow \infty} \nu\left(\Omega \backslash K_{n}\right)=0$. Also we can choose a sequence $\left(z_{n}\right) \subset \mathcal{C}_{0}(\Omega)=E$ such that $0 \leq z_{1} \leq z_{2} \leq \cdots \leq 1$ and $z_{n}\left(K_{n}\right)=1(n=1,2 \ldots)$. Then, by Corollary 3.3 , we have

$$
\begin{aligned}
\{x a y\}(\omega) & =\frac{1}{2} x(\omega)\left\{z_{n}(\bar{y} a) z_{n}\right\}+\frac{1}{2} y(\omega)\left\{z_{n}(\bar{x} a) z_{n}\right\} \\
& =\frac{1}{2} x(\omega) \int z_{n} \otimes(\bar{a} y) \otimes z_{n} d \nu_{\omega}+\frac{1}{2} y(\omega) \int z_{n} \otimes(\bar{a} x) \otimes z_{n} d \nu_{\omega} \\
& \rightarrow \frac{1}{2} x(\omega) \int 1_{\Omega} \otimes(\bar{a} y) \otimes 1_{\Omega} d \nu_{\omega}+\frac{1}{2} y(\omega) \int 1_{\Omega} \otimes(\bar{a} x) \otimes 1_{\Omega} d \nu_{\omega}
\end{aligned}
$$

where $1_{\Omega}$ denotes the function identically 1 on $\Omega$. Thus with the measure

$$
\mu_{\omega}(X):=\nu_{\omega}(\Omega \times X \times \Omega) \quad(X \text { Borel } \subset \Omega)
$$

we have the stated relation for $x, y \in E$ with compact support. The statement follows by the uniform density in $E$ of functions with compact support.

3.7. Remark. It would be tempting to conjecture that every partial $J B^{*}$-triple satisfying the hypothesis of Theorem 3.5 is the canonical $J B^{*}$ triple of some CRD. However, there is a counterexample even in two dimensions.

Let $\Omega:=\{1,2\}, \Omega_{0}:=\{1\}$ and

$$
\{x a y\}:=[1 \mapsto x(1) \overline{a(1)} y(1), 2 \mapsto x(1) \overline{a(1)} y(2) / 2+x(2) \overline{a(1)} y(1) / 2] .
$$

$\left({ }^{1}\right)$ This fact is implicit in [20]. For the sake of completeness, we include a short direct proof in the Appendix. 
Then any CRD $D$ over $\Omega$ such that the vector fields $[a-\{x a x\}] \partial / \partial x$ are complete in $D$ must be an ellipsoid of the form $D=\left\{x:|x(1)|^{2}+\lambda|x(1)|^{2}<1\right\}$ for some $\lambda>0$ with $E_{D}=E \neq E_{0}$.

4. Extension from the symmetric part. Next we are going to study partial Jordan *-triples with triple product of the form obtained in Theorem 3.5 for canonical $J B^{*}$-triples of CRDs. With the aid of bidual embedding, a technique not yet available for general partial $J B^{*}$-triples, in [17] we have achieved a finer analog of Theorem 3.5 in the special case of symmetric CRDs. Applying [17, Theorem 2] to the restriction of the triple product to the symmetric part $E_{0}$ in Theorem 3.5 , we see that the measures $\mu_{\omega}$, $\omega \in \Omega_{0}$, have finite support. Moreover, there exists a partition $\left\{\Omega_{i}: i \in I\right\}$ of $\Omega_{0}$ consisting of finite sets along with a function $m: \Omega_{0} \rightarrow \mathbb{R}$ such that, by writing $i(\omega)$ for the unique $i \in I$ with $\omega \in \Omega_{i}$, we have

$$
\mu_{\omega}=\sum_{\eta \in \Omega_{i(\omega)}} m(\eta) \delta_{\eta}, \quad \omega \in \Omega_{0}, \quad 0<\inf m \leq \sup _{i \in I} \sum_{\eta \in \Omega_{i}} m(\eta)<\infty .
$$

4.2. TheOREM. Let $\left(E, E_{0},\{\ldots\}\right)$ be a partial Jordan ${ }^{*}$-triple where $E:=\mathcal{C}_{0}(\Omega)$ with a locally compact topological space $\Omega, E_{0}:=\{f \in E$ : $\left.f\left(\Omega \backslash \Omega_{0}\right)=0\right\}$ with a non-empty open subset $\Omega_{0} \subset \Omega$, and let the triple product $\{\ldots\}$ have the form (3.6). If the measures $\mu_{\omega}$ are all positive and (4.1) holds then $\left(E, E_{0},\{\ldots\}\right)$ is a subtriple of the canonical partial JB*triple of some CRD.

Proof. By assumption, the triple product $\{\ldots\}$ satisfies axioms $(\mathrm{J} 1)-(\mathrm{J} 3)$ in Section 2. Thus to see that $\left(E, E_{0},\{\ldots\}\right)$ is a partial $J B^{*}$-triple, we have to verify axioms (J4) and (J5). According to [17, Theorem 2], the restriction of $\{\ldots\}$ to the symmetric part $E_{0} \times E_{0} \times E_{0}$ is the canonical $J B^{*}$-triple product of the symmetric CRD $D_{0}:=\left\{f \in E_{0}: \sup _{i \in I} \sum_{\eta \in \Omega_{i}} m(\eta)|f(\eta)|^{2}<1\right\}$ in $E_{0}$. Hence $\inf _{\|a\|_{\infty}=1}\|L(a) a\|_{\infty}>0$. Consider the set

$$
B:=\left\{f \in E:[\inf m] \max |f|^{2}<1, \sup _{i \in I} \sum_{\eta \in \Omega_{i}} m(\eta)|f(\eta)|^{2}<1\right\} .
$$

This is clearly a bounded convex CRD in $E$. We have

$$
B=\bigcap_{\omega \in \Omega \backslash \Omega_{0}} B_{\omega} \cap \bigcap_{i \in I} B^{(i)}
$$

with the (unbounded) CRDs $B_{\omega}:=\left\{f \in E: \mid f(\omega \mid<1 / \inf m\}\right.$ and $B^{(i)}:=$ $\left\{f \in E:\left\langle\left[f \mid \Omega_{i}\right] \mid\left[f \mid \Omega_{i}\right]\right\rangle\langle 1\}\right.$ where $\langle\cdot \mid \cdot\rangle_{i}$ denotes the scalar product $\langle\varphi \mid \psi\rangle_{i}:=\sum_{\eta \in \Omega_{i}} m(\eta) \varphi(\eta) \bar{\psi}(\eta)$. Given any function $a \in E_{0}$, we have

$$
L(a) x(\omega)=\{\operatorname{aax}\}(\omega)=\frac{1}{2} x(\omega) \int_{\eta \in \Omega_{0}}|a(\eta)|^{2} d \mu_{\omega}(\eta), \quad \omega \in \Omega \backslash \Omega_{0} ;
$$




$$
L(a) x \mid \Omega_{i}=\frac{1}{2}\left\langle\left[a \mid \Omega_{i}\right] \mid\left[a \mid \Omega_{i}\right]\right\rangle_{i}\left[x \mid \Omega_{i}\right]+\frac{1}{2}\left\langle\left[x \mid \Omega_{i}\right] \mid\left[a \mid \Omega_{i}\right]\right\rangle_{i}\left[a \mid \Omega_{i}\right], \quad i \in I .
$$

For any fixed $\omega \in \Omega \backslash \Omega_{0}$ and $\tau \in \mathbb{C}$,

$$
\exp (i \tau L(a)) x(\omega)=e^{i \tau \int|a|^{2} d \mu_{\omega}} x(\omega) .
$$

As a consequence, $\exp (i \tau L(a)) B_{\omega} \subset B_{\omega}$ whenever $\operatorname{Im} \tau \geq 0$. Similarly, $\exp (i \tau L(a)) B^{(i)} \subset B^{(i)}$ for $\operatorname{Im} \tau \geq 0$ and $i \in I$ because any mapping $\varphi \mapsto \frac{1}{2}\langle\alpha \mid \alpha\rangle_{i} \varphi+\frac{1}{2}\langle\varphi \mid \alpha\rangle_{i} \alpha$ is a positive linear operator with respect to the scalar product $\langle\cdot \mid \cdot\rangle_{i}$. Therefore $\exp (i \tau L(a)) B \subset B$ if $\operatorname{Im} \tau \geq 0$. Hence axioms $(\mathrm{J} 4)$ and $(\mathrm{J} 5)$ are immediate. Thus $\left(E, E_{0},\{\ldots\}\right)$ is a partial $J B^{*}$ triple.

Due to the form (3.6) of the triple product, the group $\Psi$ of multiplications with functions of modulus 1 consists of automorphisms of $\left(E, E_{0},\{\ldots\}\right)$. In view of Lemma 2.2 we conclude that $\left(E, E_{0},\{\ldots\}\right)$ is a subtriple of the canonical triple of some bounded domain which is invariant under multiplications with continuous functions of modulus 1 , that is, a CRD.

We can also apply the structural descriptions of Section 3 together with Theorem 4.2 to test if all inner derivations of the canonical partial $J B^{*}$ triple of a CRD can be extended in a uniformly continuous manner from the symmetric part to the whole space. As we shall see, this category does not give any counterexample.

4.3. TheOREM. On the space $E:=\mathcal{C}_{0}(\Omega)$, let $\left(E, E_{0},\{\ldots\}\right)$ be a partial $J B^{*}$-triple such that $\{\psi \cdot: \psi \in \mathcal{C}(\Omega),|\psi|=1\} \subset \operatorname{Aut}\left(E, E_{0},\{\ldots\}\right)$. Then there exists a finite constant $M$ such that $\|\Delta\| \leq M\left\|\Delta \mid E_{0}\right\|$ for all inner derivations $\Delta$ of $\left(E, E_{0},\{\ldots\}\right)$.

Proof. We know there exists an open subset $\Omega_{0}$ of $\Omega$ with $E_{0}=\{f \in$ $\left.\mathcal{C}_{0}\left(\Omega_{0}\right): f\left(\Omega \backslash \Omega_{0}\right)=0\right\}$. Furthermore, for any $\omega \in \Omega$, there is a positive Radon measure $\mu_{\omega}$ on $\Omega_{0}$ such that

$$
\{x a x\}(\omega)=x(\omega) \int_{\Omega_{0}} x \bar{a} d \mu_{\omega}, \quad x \in E, a \in E_{0}, \omega \in \Omega .
$$

Since $\mathcal{C}_{0}\left(\Omega_{0}\right)=\left\{x \bar{a} \mid \Omega_{0}: x \in E, a \in E_{0}\right\}$, the function $\omega \mapsto \int_{\Omega_{0}} f d \mu_{\omega}$ is necessarily continuous for all fixed $f \in \mathcal{C}_{0}\left(\Omega_{0}\right)$. Finally, we may assume the measures $\mu_{\omega}, \omega \in \Omega_{0}$, to be of the form (4.1). Thus, by writing $S(\omega):=\Omega_{i(\omega)}$ for short, we have

$$
\int_{\Omega_{0}} f d \mu_{\omega}=\sum_{\eta \in S(\omega)} m(\eta) f(\eta), \quad \omega \in \Omega_{0}, f \in \mathcal{C}_{0}\left(\Omega_{0}\right) .
$$

Notice also that $\omega \in S(\omega)$ for all $\omega \in \Omega_{0}$ and $0<\inf m \leq \sup m<\infty$ and $\sup _{\omega \in \Omega_{0}} \# S(\omega)<\infty$. Consider an inner derivation $\Delta$ of $\left(E, E_{0},\{\ldots\}\right)$. 
That is,

$$
\Delta x=\sum_{k=1}^{N}\left\{a_{k} b_{k} x\right\}, \quad x \in E,
$$

for some finite sequence $a_{1}, b_{1}, \ldots, a_{N}, b_{N} \in E_{0}$. In particular, given any function $x \in E=\mathcal{C}_{0}(\Omega)$,

$$
\begin{aligned}
2 \Delta x(\omega) & =\sum_{\eta \in S(\omega)} m(\eta) \sum_{k=1}^{N}\left[a_{k}(\eta) \overline{b_{k}(\eta)} x(\omega)+x(\eta) \overline{b_{k}(\eta)} a_{k}(\omega)\right] \quad \text { for } \omega \in \Omega_{0}, \\
\Delta x(\omega) & =\int_{\Omega_{0}} \sum_{k=1}^{N} a_{k}(\zeta) \overline{b_{k}(\zeta)} d \mu_{\omega}(\zeta) x(\omega) \quad \text { for } \omega \in \Omega \backslash \Omega_{0} .
\end{aligned}
$$

The continuity $\|\{x a y\}\| \leq K\|x\|\|a\|\|y\|$ of the partial triple product implies that $\sup _{\omega \in \Omega} \mu_{\omega}\left(\Omega_{0}\right) \leq K<\infty$. Hence it suffices to see that

$$
\sup _{\zeta \in \Omega_{0}}\left|\sum_{k=1}^{N} a_{k}(\zeta) \overline{b_{k}(\zeta)}\right| \leq \frac{4\left\|\Delta \mid E_{0}\right\|}{\inf m} .
$$

To prove this inequality, fix $\zeta \in \Omega_{0}$. Since the set $S(\zeta)$ is finite, for each $\omega \in S(\zeta)$ we can find $e_{\omega} \in E_{0} \equiv \mathcal{C}_{0}\left(\Omega_{0}\right)$ such that $1=e_{\omega}(\zeta)=\sup \left|e_{\omega}(\cdot)\right|$ but $e_{\omega}(\eta)=0$ for $\zeta \neq \eta \in S(\zeta)$. Then

$$
\begin{aligned}
2 \Delta e_{\omega}=\left[\sum_{k=1}^{N} \sum_{\eta \in S(\zeta)} m(\eta) a_{k}(\eta) \overline{b_{k}(\eta)}\right] e_{\omega}+\sum_{k=1}^{N} m(\omega) \overline{b_{k}(\omega)} a_{k}, \\
2 \sum_{\omega \in S(\zeta)}\left[\Delta e_{\omega}\right](\omega)=\sum_{k=1}^{N} \# S(\zeta) \sum_{\omega \in S(\zeta)} m(\omega) a_{k}(\omega) \overline{b_{k}(\omega)} \\
+\sum_{k=1}^{N} \sum_{\omega \in S(\zeta)} m(\omega) a_{k}(\omega) \overline{b_{k}(\omega)} \\
=[\# S(\zeta)+1] \sum_{\omega \in S(\zeta)} \sum_{k=1}^{N} m(\omega) a_{k}(\omega) \overline{b_{k}(\omega)} .
\end{aligned}
$$

It follows that

$$
\begin{gathered}
m(\zeta) \sum_{k=1}^{N} \overline{b_{k}(\zeta)} a_{k}(\zeta)=2\left[\Delta e_{\zeta}\right](\zeta)-\sum_{\omega \in S(\zeta)} m(\omega) \sum_{k=1}^{N} a_{k}(\omega) \overline{b_{k}(\omega)} \\
\sum_{\omega \in S(\zeta)} m(\omega) \sum_{k=1}^{N} a_{k}(\omega) \overline{b_{k}(\omega)}=\frac{2}{\# S(\zeta)+1} \sum_{\omega \in S(\zeta)}\left[\Delta e_{\omega}\right](\omega) .
\end{gathered}
$$


Notice that $\left\|e_{\omega}\right\|=\max \left|e_{\omega}(\cdot)\right|=1$ and hence $\left|\left[\Delta e_{\omega}\right](\omega)\right| \leq\left\|\Delta \mid E_{0}\right\|$ for all $\omega \in \Omega$. Therefore

$$
m(\omega)\left|\sum_{k=1}^{N} a_{k}(\omega) \overline{b_{k}(\omega)}\right| \leq 2\left\|\Delta\left|E_{0}\left\|+\frac{2}{\# S(\zeta)+1} \sum_{\omega \in S(\zeta)}\right\| \Delta\right| E_{0}\right\| \leq 4\left\|\Delta \mid E_{0}\right\| .
$$

This completes the proof of (4.4) and hence the proof of the theorem.

5. The fine structure of the canonical partial $J B^{*}$-triple of a CRD. Throughout this section, $\Omega$ denotes a locally compact Hausdorff space, $\Omega_{0} \neq \emptyset$ is a fixed open subset of $\Omega$, and we write $E:=\mathcal{C}_{0}(\Omega)$ and $E_{0}:=\left\{f \in E: f\left(\Omega \backslash \Omega_{0}\right)=0\right\}$. Also we reserve the notations $\left[\mu_{\omega}\right.$ : $\omega \in \Omega],\left\{\Omega_{i}: i \in I\right\}$ and $m$, respectively, for a given measure-valued map $\Omega \rightarrow \mathcal{M}\left(\Omega_{0}\right)_{+}$, a partition of $\Omega_{0}$ into finite non-empty sets, and a function $m: \Omega_{0} \rightarrow \mathbb{R}_{+}$such that (4.1) holds. We know from Theorems 3.5 and 4.2 that the canonical triple product of a CRD has necessarily the form (3.6) in terms of these objects.

Our purpose will be to find a description in terms of the topological properties of the partition $\left\{\Omega_{i}: i \in I\right\}$ when a triple product of the form (3.6) is the canonical triple product of some CRD. It is clear that there are plenty of mappings $\omega \mapsto \mu_{\omega}$, even satisfying (4.1), for which the operation (3.6) is not a partial $J B^{*}$-triple product. Indeed, the following observation is an immediate but fundamental consequence of Theorem 3.5 and its proof. Given a bounded Reinhardt domain $D$ in E, the canonical triple product $\{\ldots\}:=\{\ldots\}_{D}$ has the form

$$
\{x a y\}=\frac{1}{2} x A(\bar{a} y)+\frac{1}{2} y A(\bar{a} x), \quad a \in E_{0}, x, y \in E,
$$

with some positive linear map $A: E_{0} \rightarrow \mathcal{C}_{b}(\Omega):=\{$ bounded continuous functions $\Omega \rightarrow \mathbb{C}\}$. It is well-known [11] that the positivity of $A$ entails its boundedness automatically. Notice also that, by the Riesz-Kakutani representation theorem, any positive linear mapping $A: \mathcal{C}_{0}\left(\Omega_{0}\right) \rightarrow \mathcal{C}_{b}(\Omega)$ has the form $A f(\omega)=\int_{\Omega_{0}} f d \mu_{\omega}$ with a uniquely determined mapping $\Omega \ni \omega \mapsto \mu_{\omega} \in \mathcal{M}\left(\Omega_{0}\right)_{+}$.

5.2. Lemma. Suppose $A: E_{0} \rightarrow \mathcal{C}_{b}(\Omega)$ is a positive linear mapping. Then the structure $\left(E, E_{0},\{\ldots\}\right)$ with the operation (5.1) is a partial Jordan ${ }^{*}$-triple if and only if

$$
\begin{aligned}
A(f A(g)) & =A(g A(f)), \\
A(f A(g)) \mid \Omega_{0} & =A(f) A(g) \mid \Omega_{0}, \quad f, g \in E_{0} .
\end{aligned}
$$

Proof. Since $E_{0}$ and $E$ are closed ideals in $\mathcal{C}_{b}(\Omega)$ with respect to the pointwise product of functions, the operation (5.1) is a well-defined positive 
continuous sesquitrilinear map $E \times E_{0} \times E \rightarrow E$. It satisfies the identities

$$
\begin{aligned}
& \{x a\{x b x\}\}=\frac{1}{2} x A(\bar{a} x A(\bar{b} x))+\frac{1}{2} x A(\bar{b} x) A(\bar{b} x), \\
& \left.\{x a\{x b x\}\}-\{x b\{x a x\}\}=\frac{1}{2} x A[\bar{a} x A(\bar{b} x))-\bar{b} x A(\bar{a} x)\right] .
\end{aligned}
$$

Hence, by taking $f:=\bar{a} x$ and $g:=\bar{b} x$, we see that (5.3) implies axiom (J3). Conversely, assume (J3) holds. Then $x A[\bar{a} x A(\bar{b} x))-\bar{b} x A(\bar{a} x)]=0$ for $a, b \in E_{0}$ and $x \in E$. Consider any functions $f, g \in E_{0}$ with compact support. Then, given any point $\omega \in \Omega$, we can choose a function $x_{\omega} \in E$ with compact support such that the interior of $\operatorname{supp}\left(x_{\omega}\right)$ contains $\{\omega\} \cup \operatorname{supp}(f) \cup \operatorname{supp}(g)$. Then we can write $f=\bar{a}_{\omega} x_{\omega}$ and $g=\bar{b}_{\omega} x_{\omega}$ with some $a_{\omega}, b_{\omega} \in E_{0}$ and hence (J3) implies $0=A[f A(g)-g A(f)](\omega)$. Thus, since the functions with compact support are dense in $E_{0}$, axiom (J3) is equivalent to the identity $A(f A(g))=A(g A(f))$ in (5.3).

Let us now proceed to the axiom (J2) of the Jordan identity. By polarization, (J2) is equivalent to its special case

$$
\{a a\{x b x\}\}=2\{\{a a x\} b x\}-\{x\{a a b\} x\}, \quad a, b \in E_{0}, x \in E .
$$

In terms of the operation $A$, this identity (multiplied by 2 ) can be stated as

$$
\begin{aligned}
a A(\bar{a} x A(\bar{b} x))+ & x A(\bar{b} x) A\left(|a|^{2}\right) \\
= & {\left[a A(\bar{a} x)+x A\left(|a|^{2}\right)\right] A(\bar{b} x)+x A\left(\bar{b}\left[a A(\bar{a} x)+x A\left(|a|^{2}\right)\right]\right) } \\
& -x A\left(\left[a A(\bar{a} b)+b A\left(|a|^{2}\right)\right]^{-} x\right) .
\end{aligned}
$$

By the positivity of $A$, we can write the last term above as $-x A([\bar{a} A(a \bar{b})+$ $\left.\left.\bar{b} A\left(|a|^{2}\right)\right] x\right)$. Thus, by the linearity of $A$, axiom $\left(\mathrm{J}^{\prime}\right)$ is equivalent to

$$
\begin{aligned}
a A & (\bar{a} x A(\bar{b} x))+x A(\bar{b} x) A\left(|a|^{2}\right) \\
= & a A(\bar{a} x) A(\bar{b} x)+x A\left(|a|^{2}\right) A(\bar{b} x)+x A(\bar{b} a A(\bar{a} x))+x A\left(\bar{b} x A\left(|a|^{2}\right)\right) \\
& \quad-x A(\bar{a} x A(a \bar{b}))-x A\left(\bar{b} x A\left(|a|^{2}\right)\right) .
\end{aligned}
$$

Here the terms $x A(\bar{b} x) A\left(|a|^{2}\right)$ and $x A\left(\bar{b} x A\left(|a|^{2}\right)\right)$ cancel each other, whence we get

$$
a A(\bar{a} x A(\bar{b} x))=a A(\bar{a} x) A(\bar{b} x)+x A(\bar{b} a A(\bar{a} x))-x A(\bar{a} x A(a \bar{b})) .
$$

Observe that (5.3) implies ( $\left.\mathrm{J}^{\prime \prime}\right)$ immediately. To finish the proof, assume $(\mathrm{J} 2)+(\mathrm{J} 3)$. As we have shown, this is nothing else than the identity $A(f A(g))$ $=A(g A(f))$ along with $\left(\mathrm{J}^{\prime \prime}\right)$. By substituting $f:=\bar{a} x$ and $g:=a \bar{b}$ in $\left(\mathrm{J}^{\prime \prime}\right)$, we see that two terms cancel each other and the remaining identity $a A(\bar{a} x A(\bar{b} x))=a A(\bar{a} x) A(\bar{b} x)$ is equivalent to its polarized form

$$
a_{1} A\left(\overline{a_{2}} x A(\bar{b} x)\right)=a_{1} A\left(\overline{a_{2}} x\right) A(\bar{b} x), \quad a_{1}, a_{2}, b \in E_{0}, x \in E .
$$

Since each $a_{1} \in E_{0}$ vanishes outside $\Omega_{0}$, but for any $\omega \in \Omega_{0}$ there is a func- 
tion $a_{1, \omega} \in E_{0}$ with $a_{1, \omega}(\omega) \neq 0$, the polarized identity is further equivalent to

$$
A(\bar{a} x A(\bar{b} x))\left|\Omega_{0}=A(\bar{a} x) A(\bar{b} x)\right| \Omega_{0}, \quad a, b \in E_{0}, x \in E .
$$

As we have seen, any functions $f, g \in E_{0}$ with compact support can be written in the form $f=\bar{a} x, g=\bar{b} x$ for suitable $a, b \in E_{0}$ and $x \in E$ with compact support. This implies the second identity in (5.3) for functions with compact support, and the statement follows by a standard density argument.

5.4. REMARK. An application of the results in [7] concerning symmetric CRDs to the symmetric part of the canonical partial $J B^{*}$-triple of a CRD yields the following observation. If $\left(E, E_{0},\{\ldots\}\right)$ is a partial $J B^{*}$-triple with a triple product of the form (3.6) and having property (4.1), then the setvalued function $\omega \mapsto \Omega_{i(\omega)} \cup\{\infty\}$ (where $i(\omega)$ denotes the unique $i \in I$ with $\omega \in \Omega_{i}$ ) is continuous with respect to the Hausdorff topology on the set of non-empty compact subsets of $\Omega_{0} \cup\{\infty\}$. As a consequence, given a relatively closed subset $F$ of $\Omega_{0}$ and a point $\omega \in F$ such that $\#\left[F \cap \Omega_{i(\omega)}\right]=$ $N_{F}:=\max _{\eta \in F} \#\left[F \cap \Omega_{i(\eta)}\right]$, there are disjoint open sets $U_{1}, \ldots, U_{N_{F}} \subset \Omega_{0}$ such that $\omega \in U_{1}$ and $\#\left[U_{k} \cap F \cap \Omega_{i(\eta)}\right]=1$ for any $\eta \in U_{1} \cup \cdots \cup U_{N_{F}}$ and $k=1, \ldots, N_{F}$.

5.5. Lemma. Assume the mapping $\omega \mapsto \Omega_{i(\omega)} \cup\{\infty\}$ is Hausdorff continuous in the sense of 5.4. Then given any $\omega \in \Omega$, there exists a finite family of disjoint Borel subsets $G_{1}, \ldots, G_{N} \subset \Omega_{0}$ such that $\mu_{\omega}\left(\Omega_{0} \backslash \bigcup_{k=1}^{N} G_{k}\right)=0$ and $\#\left[\Omega_{i} \cap G_{k}\right] \leq 1$ for all $i \in I$ and $k=1, \ldots, N$.

Proof. We use countable transfinite exhaustion to construct the sets $G_{1}, \ldots, G_{N}$. To start, let $N:=N_{\Omega_{0}}, F^{(0)}:=\Omega_{0}$ and $U_{1}^{(0)}, \ldots, U_{N}^{(0)}:=\emptyset$. For any countable ordinal $r \succ 0$, if until each set $U_{k}^{(s)}$ with $s \prec r$ and $1 \leq k \leq N$ is open and $\mu_{\omega}\left(\bigcup_{s \prec r} \bigcup_{k=1}^{N} U_{k}^{(s)}\right)<\mu_{\omega}\left(\Omega_{0}\right)$, define $F^{(r)}:=$ $\Omega_{0} \backslash \bigcup_{s \prec r} \bigcup_{k=1}^{N} U_{k}^{(s)}, N_{r}:=\max _{\eta \in F^{(r)}} \#\left[F^{(r)} \cap \Omega_{i(\eta)}\right]$. We also choose $\omega_{r} \in F^{(r)}$ with $\#\left[F^{(r)} \cap \Omega_{i\left(\omega_{r}\right)}\right]=N_{r}$ along with a finite disjoint family $U_{1}^{(r)}, \ldots, U_{N_{r}}^{(r)}$ such that $\mu_{\omega}\left(U_{1}^{(r)}\right)>0$ and $\#\left[U_{k}^{(r)} \cap \Omega_{i(\eta)}\right]=1$ for all $\eta \in U_{1}^{(r)} \cup \cdots \cup U_{N_{r}}^{(r)}$ and $k=1, \ldots, N_{r}$. Finally, we set $U_{k}^{r}:=\emptyset$ for $N_{r}<k \leq N$. This can be done in view of Remark 5.4 and the fact that trivially $N_{r} \leq N$. Since the measure $\mu_{\omega}$ is finite, in this manner, for some countable ordinal $r^{*}$, we get a family $\left\{U_{k}^{(r)}: r \prec r^{*}, k=1, \ldots, N\right\}$ of open subsets of $\Omega_{0}$ such that $\mu_{\omega}\left(\Omega_{0} \backslash \bigcup_{s \prec r^{*}} \bigcup_{k=1}^{N} U_{k}^{(s)}\right)=0$ and $\#\left[U_{k}^{(r)} \cap F^{(r)} \cap \Omega_{i(\eta)}\right] \leq 1$, $1 \leq k \leq N$, but $\Omega_{i(\eta)} \subset \bigcup_{k=1}^{N}\left[U_{k}^{(r)} \cap F^{(r)}\right]$ for all $\eta \in \bigcup_{k=1}^{N}\left[U_{k}^{(r)} \cap F^{(r)}\right]$ for all ordinals $r \prec r^{*}$. Therefore the choice $G_{k}:=\bigcup_{s \prec r^{*}}\left[U_{k}^{(s)} \cap F^{(s)}\right], k=1, \ldots, N$, suits our requirements. 
5.6. Corollary. Let $\mathcal{K}:=\left\{K \subset I: \bigcup_{i \in K} \Omega_{i}\right.$ is Borel measurable $\}$ and define $\widetilde{\mu}_{\omega}(K):=\mu_{\omega}\left(\bigcup_{i \in K} \Omega_{i}\right), K \in \mathcal{K}$. Then (under the hypothesis of Lemma 5.5) there is a Borel function $p_{\omega}: \Omega_{0} \rightarrow[0,1]$ such that $\sum_{\eta \in \Omega_{i}} p_{\omega}(\eta)=1, i \in I$, and for all bounded Borel functions $f: \Omega_{0} \rightarrow \mathbb{C}$ we have

$$
\int_{\Omega_{0}} f d \mu_{\omega}=\int_{i \in I} \sum_{\eta \in \Omega_{i}} f(\eta) p_{\omega}(\eta) d \widetilde{\mu}_{\omega}(i) .
$$

Proof. As already noted, the sets

$$
G_{k}^{(r)}:=U_{k}^{(r)} \cap F^{(r)}=U_{k}^{(r)} \backslash \bigcup_{s \prec r} \bigcup_{k=1}^{N_{r}} U_{k}^{(s)}, \quad r \prec r^{*}, 1 \leq k \leq N_{r},
$$

form a disjoint covering of $\Omega_{0}$ up to a set of $\mu_{\omega}$-measure 0 . Let $\widetilde{p}_{k}^{(r)}$ denote the Radon-Nikodým derivative $d \widetilde{\mu}_{\omega, k}^{(r)} / d \widetilde{\mu}_{\omega}$ with the measure $\widetilde{\mu}_{\omega, k}^{(r)}(K):=$ $\mu_{\omega}\left(G_{k}^{(r)} \cap \bigcup_{i \in K} \Omega_{i}\right), K \in \mathcal{K}$. These are functions $I \rightarrow \mathbb{R}$ defined up to a set of $\widetilde{\mu}_{\omega}$-measure 0 , and we can choose Borel measurable representatives with $0 \leq \widetilde{p}_{k}^{(r)} \leq 1$ and $\sum_{k=1}^{N_{r}} \widetilde{p}_{k}^{(r)}=1$ on $I^{(r)}:=\left\{i \in I: \Omega_{i} \subset \bigcup_{k=1}^{N_{r}} G_{k}^{(r)}\right\}$ and vanishing outside $I^{(r)}$. This can be done because every $\Omega_{i}$ meets any set $G_{k}^{(r)}$ in at most one point and for the sets $G^{(r)}:=\bigcup_{k=1}^{N_{r}} G_{k}^{(r)}$ we have either $\Omega_{i} \subset G^{(r)}$ or $\Omega_{i} \cap G^{(r)}=\emptyset$. Hence the statement holds with the function $p(\eta):=\sum_{r \prec r^{*}} \sum_{k=1}^{N_{r}} \widetilde{p}_{k}^{(r)}(i(\eta)), \eta \in \Omega_{0}$.

5.7. Corollary. Suppose we have (4.1) with a weight function $m>0$ and let $A: \mathcal{C}_{0}\left(\Omega_{0}\right) \rightarrow \mathcal{C}_{b}(\Omega)$ have the form $A f(\omega)=\int_{\eta \in \Omega_{0}} f d \mu_{\omega}$ with suitable Radon measures $\mu_{\omega}, \omega \in \Omega$. Then the identity $A(f A(g))=A(g A(f))$ is equivalent to the fact that

$$
\mu_{\omega}(X)=\int_{i \in I} \sum_{\eta \in X \cap \Omega_{i}} m(\eta) d \kappa_{\omega}(i), \quad X \subset \Omega_{0},
$$

with suitable measures $\kappa_{\omega}:\left\{K \subset I: \bigcup_{i \in K} \Omega_{i}\right.$ is Borel measurable $\} \rightarrow \mathbb{R}_{+}$, $\omega \in \Omega$.

Proof. Using Corollary 5.6, we can write

$$
\begin{aligned}
{[A(f A(g))](\omega) } & =\int_{i \in I} \sum_{\eta \in \Omega_{i}} f(\eta)[A(g)](\eta) p_{\omega}(\eta) d \widetilde{\mu}_{\omega}(i) \\
& =\int_{i \in I} \sum_{\eta \in \Omega_{i}} f(\eta) \sum_{\zeta \in \Omega_{i(\eta)}} g(\zeta) m(\zeta) p_{\omega}(\eta) d \widetilde{\mu}_{\omega}(i) \\
& =\int_{i \in I} \sum_{\zeta, \eta \in \Omega_{i}} f(\eta) g(\zeta) m(\zeta) p_{\omega}(\eta) d \widetilde{\mu}_{\omega}(i)
\end{aligned}
$$

because $i(\eta)=i$ for $\eta \in \Omega_{i}$. Thus the identity $A(f A(g))=A(g A(f))$ is 
equivalent to

$$
0=\int_{i \in I} \sum_{\zeta, \eta \in \Omega_{i}} f(\eta) g(\zeta)\left[m(\zeta) p_{\omega}(\eta)-m(\eta) p_{\omega}(\zeta)\right] d \widetilde{\mu}_{\omega}(i)
$$

for all $f, g \in \mathcal{C}_{0}\left(\Omega_{0}\right)$ and $\omega \in \Omega$. By passing to limits of monotone sequences, we see that (5.9) holds for all $f, g \in \mathcal{C}_{0}\left(\Omega_{0}\right)$ if and only if it holds for all bounded Borel measurable functions $f, g: \Omega_{0} \rightarrow \mathbb{C}$. Consider (5.9) for the partition $\Omega_{0}=\bigcup_{r \prec r^{*}} \bigcup_{k=1}^{N_{r}} G_{k}^{(r)} \cup\left[\mu_{\omega^{-}}\right.$zero-set $]$constructed in the proof of Corollary 5.6. By writing $\zeta_{i, k}^{(r)}$ for the unique element of $\Omega_{i} \cap G_{k}^{(r)}$, we get

$$
\begin{aligned}
0=\sum_{r \prec r^{*}} \sum_{k, l=1}^{N_{r}} \int_{i \in I^{(r)}} f\left(\zeta_{i, k}^{(r)}\right) g\left(\zeta_{i, l}^{(r)}\right)\left[m\left(\zeta_{i, k}^{(r)}\right) p_{\omega}\left(\zeta_{i, l}^{(r)}\right)\right. \\
\left.-m\left(\zeta_{i, l}^{(r)}\right) p_{\omega}\left(\zeta_{i, k}^{(r)}\right)\right] d \widetilde{\mu}_{\omega}(i) .
\end{aligned}
$$

This holds for all bounded Borel functions $f, g: \Omega_{0} \rightarrow \mathbb{C}$ if and only if, given any $r \prec r^{*}$, for $\widetilde{\mu}_{\omega}$-almost every $i \in I^{(r)}$ we have

$$
m\left(\zeta_{i, k}^{(r)}\right) p_{\omega}\left(\zeta_{i, l}^{(r)}\right)-m\left(\zeta_{i, l}^{(r)}\right) p_{\omega}\left(\zeta_{i, k}^{(r)}\right)=0, \quad 1 \leq k, l \leq N_{r}
$$

Indeed, if we just consider functions $f, g$ vanishing outside $G_{k}^{(r)}$ respectively $G_{l}^{(r)}$ (with fixed $r \prec r^{*}$ and $1 \leq k, l \leq N_{r}$ ), we obtain $\left(5.9^{\prime}\right)$ without the summations $\sum_{r \prec r^{*}}$ and $\sum_{k, l=1}^{N_{r}}$, whence the statement is immediate. Thus, since $\sum_{\zeta \in \Omega_{i}} p_{\omega}(\zeta)=1$ for $\widetilde{\mu}_{\omega}$-almost every $i \in I,\left(5.9^{\prime}\right)$ holds for all bounded Borel functions if and only if

$$
p_{\omega}(\eta)=m(\eta)\left[\sum_{\zeta \in \Omega_{i}} m(\zeta)\right]^{-1} \quad \text { for } \widetilde{\mu} \text {-almost every } i \in I \text { and } \eta \in \Omega_{i} \text {. }
$$

This observation establishes the statement of 5.7 with the measures $\kappa_{\omega}(K)$ $:=\int_{i \in K}\left[\sum_{\zeta \in \Omega_{i}} m(\zeta)\right]^{-1} d \widetilde{\mu}_{\omega}(i), K \in \mathcal{K}$.

6. Bidual of the canonical $J B^{*}$-triple of a CRD. On the basis of the previous section, first we give an exhaustive parametric description of the canonical $J B^{*}$-triples of continuous Reinhardt domains. Also we answer in the affirmative the question if the bidual of the canonical $J B^{*}$-triple of a continuous Reinhardt domain can be regarded as the canonical $J B^{*}$-triple of a continuous Reinhardt domain in the bidual commutative $C^{*}$-algebra.

As in the previous sections, $\Omega$ denotes a fixed locally compact Hausdorff space, $\Omega_{0}$ is a non-empty open subset of $\Omega, m$ is a function $\Omega_{0} \rightarrow \mathbb{R}$ and $\Pi=\left\{\Omega_{i}: i \in I\right\}$ is a partition of $\Omega_{0}$. We shall write $\Omega_{0} / \Pi$ for the index set $I$ of $\Pi$ equipped with the topology inherited from the Hausdorff topology of $\widetilde{\Omega}_{0}:=\left\{\Omega_{i} \cup\{\infty\}: i \in I\right\}$. That is, a set $J \subset I$ is open if $\left\{\Omega_{i} \cup\{\infty\}: i \in J\right\}$ is an open subset of $\widetilde{\Omega}_{0}$ with respect to the Hausdorff topology of the compact subsets on $\Omega_{0} \cup\{\infty\}$ restricted to $\widetilde{\Omega}_{0}$. 
6.1. Definition (cf. $[7,1.1-2]$ ). We say that the couple $(m, \Pi)$ is admissible if $\sup _{i \in I} \# \Omega_{i}<\infty, 0<\inf m \leq \sup m<\infty$ and all the functions $\Omega_{0} \ni \omega \mapsto \sum_{\eta \in \Omega_{i(\omega)}} m(\eta) f(\eta), f \in \mathcal{C}_{0}\left(\Omega_{0}\right)$, are continuous.

According to $[7,1.2]$, the couple $(m, \Pi)$ is admissible if and only if the function space $\mathcal{C}_{0}\left(\Omega_{0}\right)$ endowed with the triple product polarized from $\{x a x\}(\omega):=\sum_{\zeta \in \Omega_{i(\omega)}} m(\zeta) x(\zeta) \overline{a(\zeta)} x(\omega)$ (where $i(\omega)$ denotes the (unique) index with $\left.\omega \in \Omega_{i(\omega)}\right)$ is the canonical triple of some symmetric Reinhardt domain in $\mathcal{C}_{0}\left(\Omega_{0}\right)$. Furthermore, as a conseqence of [7, 1.3(iii)], given an admissible couple $(m, \Pi)$, the topological space $\Omega_{0} / \Pi$ is locally compact and Hausdorff.

6.2. Lemma. Let $(m, \Pi)$ be an admissible couple.

(1) A function $\phi: I \rightarrow \mathbb{C}$ belongs to $\mathcal{C}_{0}\left(\Omega_{0} / \Pi\right)$ if and only if the function $f_{\phi}:=[\omega \mapsto \phi(i(\omega))]$ is bounded, continuous on $\Omega_{0}$, constant on the sets $\Omega_{i}, i \in I$, and such that for any $\varepsilon>0$ there exists a compact subset $K_{\varepsilon} \subset \Omega_{0}$ with $\left|f_{\phi}\left(\Omega_{i}\right)\right|<\varepsilon$ whenever $\Omega_{i} \cap K_{\varepsilon}=\emptyset$.

(2) The range of the operator $\widetilde{A}_{0}$ on $\mathcal{C}_{0}\left(\Omega_{0}\right)$ defined by

$$
\widetilde{A}_{0} f(i):=\sum_{\zeta \in \Omega_{i(\omega)}} m(\zeta) f(\zeta), \quad i \in I, f \in \mathcal{C}_{0}\left(\Omega_{0}\right),
$$

is a uniformly dense multiplicative ideal in $\mathcal{C}_{0}\left(\Omega_{0} / \Pi\right)$.

Proof. (1) Let $\phi \in \mathcal{C}_{0}\left(\Omega_{0} / \Pi\right)$. By construction, $f_{\phi}$ is constant on each $\Omega_{i}$. Also the ranges of $\phi$ and $f_{\phi}$ coincide, thus $f_{\phi}$ is necessarily bounded. Consider a convergent net $\omega_{j} \rightarrow \omega_{0}$ in $\Omega_{0}$. According to [7, 1.2(iv)], we have $\Omega_{i\left(\omega_{j}\right)} \cup\{\infty\} \rightarrow \Omega_{i\left(\omega_{0}\right)} \cup\{\infty\}$ with respect to the Hausdorff topology. Therefore $f_{\phi}\left(\omega_{j}\right) \rightarrow f_{\phi}\left(\omega_{0}\right)$, showing that $f_{\phi} \in \mathcal{C}_{b}\left(\Omega_{0}\right)$. The stated vanishing property of $f_{\phi}$ at infinity is straightforward. Conversely, assume that $\phi: I \rightarrow \mathbb{C}$ is such that $f_{\phi} \in \mathcal{C}_{b}\left(\Omega_{0}\right)$ with the behavior at infinity as in (1). Then $\phi$ vanishes at infinity in the sense of the locally compact inherited Hausdorff topology of $\Omega_{0} / \Pi$. We show the continuity of $\phi$ as follows. Let $\left[i_{j}: j \in J\right]$ be a net in $I$ such that $\Omega_{i_{j}} \cup\{\infty\} \rightarrow \Omega_{i_{0}} \cup\{\infty\}$ in the Hausdorff sense. By [7, 1.3(i)] we can find a convergent net $\omega_{j} \rightarrow \omega_{0}$ in $\Omega_{0}$ with $\omega_{j} \in \Omega_{i_{j}}, j \in J$ and $\omega_{0} \in \Omega_{i_{0}}$. Hence $\phi\left(i_{j}\right)=f_{\phi}\left(\Omega_{i_{j}}\right)=f_{\phi}\left(\omega_{j}\right) \rightarrow f_{\phi}\left(\omega_{0}\right)=f_{\phi}\left(\Omega_{i_{0}}\right)=\phi\left(i_{0}\right)$.

(2) As already noted, for each $f \in \mathcal{C}_{0}\left(\Omega_{0}\right)$ the function $A_{0} f:=\left[\Omega_{0} \ni \omega\right.$ $\left.\mapsto \sum_{\zeta \in \Omega_{i(\omega)}} m(\zeta) f(\zeta)\right]$ is continuous. Obviously, $A_{0} f$ is constant on each $\Omega_{i}$. Given a net $\left[i_{j}: j \in J\right]$ such that $\Omega_{i_{j}} \rightarrow\{\infty\}$ in the Hausdorff sense (i.e. for every $K$ compact $\subset \Omega_{0}$ there exists $j_{K} \in J$ with $K \cap \Omega_{i_{j}}=\emptyset$ for $j \geq j_{K}$ ), we have $A_{0} f\left(\Omega_{i_{j}}\right) \rightarrow 0$ because

$$
\left|A_{0} f\left(\Omega_{i_{j}}\right)\right| \leq \sup _{\omega} m(\omega) \max _{i} \# \Omega_{i} \max _{\zeta \in \mathrm{V}}|f(\zeta)|
$$


and $\max _{\zeta \in \mathrm{V}}|f(\zeta)| \rightarrow 0$. By (1), $A_{0} f=f_{\phi}$ for some $\phi \in \mathcal{C}_{0}\left(\Omega_{0} / \Pi\right)$. Thus $\operatorname{ran} \widetilde{A}_{0} \subset \mathcal{C}_{0}\left(\Omega_{0} / \Pi\right)$. Observe that, for any $\psi \in \mathcal{C}_{0}\left(\Omega_{0} / \Pi\right)$ we have $\psi\left[\widetilde{A}_{0} f\right]=$ $\widetilde{A}_{0}\left(f_{\psi} f\right)$. Thus ran $\widetilde{A}_{0}$ is an ideal in $\mathcal{C}_{0}\left(\Omega_{0} / \Pi\right)$. For any $i \in I$, there exists $\phi \in \operatorname{ran} \widetilde{A}_{0}$ with $\phi(i) \neq 0$. Indeed, by choosing any element $\omega \in \Omega_{i}$, there exists a function $f \in \mathcal{C}_{0}\left(\Omega_{0}\right)$ with $f(\omega)=1$ and $f(\zeta)=0$ for $\zeta \in \Omega_{i} \backslash\{\omega\}$ and $\widetilde{A}_{0} f(i)=\sum_{\zeta \in \Omega_{i}} m(\zeta) f(\zeta)=m(\omega) f(\omega)>0$. Hence, by the StoneWeierstrass theorem, the ideal $\operatorname{ran} \widetilde{A}_{0}$ is uniformly dense in $\mathcal{C}_{0}\left(\Omega_{0} / \Pi\right)$.

6.4. Definition. Given an admissible couple $(m, \Pi)$ and a non-negative measure-valued mapping $\omega \mapsto \kappa_{\omega}$ from $\Omega$ to $\mathcal{M}(\Omega / \Pi)$, write

$$
\mathbf{E}\left(\Omega, \Omega_{0}, m, \Pi, \kappa\right)
$$

for the structure $\left(\mathcal{C}_{0}(\Omega),\left\{f \in \mathcal{C}_{0}(\Omega): f\left(\Omega \backslash \Omega_{0}\right)=0\right\},\{\ldots\}\right)$ where the triple product $\{\ldots\}$ is the polarized form of

$$
\begin{aligned}
\{x a x\} & (\omega)=x(\omega) \int_{i \in I} \sum_{\zeta \in \Omega_{i}} m(\zeta) \overline{a(\zeta)} x(\zeta) d \kappa_{\omega}(i), \\
\omega & \in \Omega, a \in E_{0}, x \in E .
\end{aligned}
$$

We say that the tuple $\left(\Omega, \Omega_{0}, m, \Pi, \kappa\right)$ is admissible if $(m, \Pi)$ is an admissible couple and the measure-valued mapping $\omega \mapsto \kappa_{\omega}$ is weakly continuous $\left({ }^{2}\right)$ and such that $\kappa_{\omega}=\delta_{i(\omega)}$ whenever $\omega \in \Omega_{0}$.

6.6. Theorem. Let $\Omega$ be a locally compact Hausdorff space and $\emptyset \neq$ $\Omega_{0} \subset \Omega$ an open subset. By setting $E:=\mathcal{C}_{0}(\Omega), E_{0}:=\left\{f \in E: f\left(\Omega \backslash \Omega_{0}\right)=\right.$ $0\}$ the triple $\left(E, E_{0},\{\ldots\}\right)$ is a subtriple of the canonical $J B^{*}$-triple of some Reinhardt domain in $E$ if and only if it is of the form $\mathbf{E}\left(\Omega, \Omega_{0}, m, \Pi, \kappa\right)$ with an admissible tuple $\left(\Omega, \Omega_{0}, m, \Pi, \kappa\right)$.

The canonical $J B^{*}$-triple of any Reinhardt domain in $\mathcal{C}_{0}(\Omega)$ with nonzero symmetric part has the form $\mathbf{E}\left(\Omega, \Omega_{0}, m, \Pi, \kappa\right)$ with a suitable admissible tuple $\left(\Omega, \Omega_{0}, m, \Pi, \kappa\right)$.

Proof. We already know the following facts from Theorem 3.5 and Corollary 5.7. The canonical $J B^{*}$-triple of any Reinhardt domain with non-zero symmetric part in $E:=\mathcal{C}_{0}(\Omega)$ coincides with $\mathbf{E}\left(\Omega, \Omega_{0}, m, \Pi, \kappa\right)$ for some open $\emptyset \neq \Omega_{0} \subset \Omega$ and an admissible couple $(\Pi, m)$. Moreover any partial Jordan *-triple $\left(E, E_{0},\{\ldots\}\right)$ with $E_{0}=\left\{f \in E: f\left(\Omega \backslash \Omega_{0}\right)=0\right\}$ for some $\emptyset \neq \Omega_{0} \subset \Omega$ and such that all multiplications with continuous functions $\Omega \rightarrow \mathbb{T}(=\{z \in \mathbb{C}:|z|=1\})$ belong to $\operatorname{Aut}\left(E, E_{0},\{\ldots\}\right)$ must have the form $\mathbf{E}\left(\Omega, \Omega_{0}, m, \Pi, \kappa\right)$ with suitable open $\emptyset \neq \Omega_{0} \subset \Omega$ and an admissible couple $(\Pi, m)$. Finally, by Lemma 2.2 and Corollary 5.7 , each $\mathbf{E}\left(\Omega, \Omega_{0}, m, \Pi, \kappa\right)$ is a subtriple of the canonical $J B^{*}$-triple of some Reinhardt domain in $E$ if and only if the triple product maps $E \times E_{0} \times E$ to $E$.

$\left({ }^{2}\right)$ That is, $\omega \mapsto \int_{i \in I} \phi(i) d \kappa_{\omega}(i)$ is continuous for every $\phi \in \mathcal{C}_{0}(\Omega / \pi)$. 
Thus it remains to prove that, in a structure of the form $\mathbf{E}\left(\Omega, \Omega_{0}, m, \Pi, \kappa\right)$, the triple product maps $E \times E_{0} \times E$ into $E$ (where $E:=\mathcal{C}_{0}(\Omega)$ and $\left.E_{0}:=\left\{f \in E: f\left(\Omega \backslash \Omega_{0}\right)=0\right\}\right)$ if and only if the mapping $\omega \mapsto \kappa_{\omega}$ is weakly continuous. The sufficiency of the weak continuity of $\kappa$ for $\left\{E E_{0} E\right\} \subset E$ is immediate. Conversely, suppose $(m, \Pi)$ is an admissible couple and the triple product $(6.5)$ is continuous and satisfies $\left\{E E_{0} E\right\} \subset E$. Then, by Corollary $5.7,\left(E, E_{0},\{\ldots\}\right)=\mathbf{E}\left(\Omega, \Omega_{0}, m, \Pi, \kappa\right)$ is a partial $J B^{*}$-triple and, in particular, the operation

$$
A f(\omega)=\int_{\eta \in \Omega_{0}} \sum_{\zeta \in \Omega_{i(\eta)}} m(\zeta) f(\zeta) d \mu_{\omega}(\eta)=\int \widetilde{A}_{0} f d \kappa_{\omega}, \quad \omega \in \Omega, f \in E_{0},
$$

has values in the space $\mathcal{C}_{b}(\Omega)$ of all bounded continuous functions $\Omega \rightarrow \mathbb{C}$. Therefore also the operation $T_{0} g:=\left[\Omega \ni \omega \mapsto \int \psi d \kappa_{\omega}\right], \psi \in \operatorname{ran} \widetilde{A}_{0}$, has values in $\mathcal{C}_{b}(\Omega)$. We know that the measures $\mu_{\omega}, \omega \in \Omega$, have total mass bounded by the norm $M:=\sup _{a \in E_{0}, x, y \in E}\|\{x a y\}\|$ of the triple product. It follows that $\kappa_{\omega}(I) \leq M, \omega \in \Omega$, and hence $T_{0}$ is bounded with norm $\leq M$ (i.e. $\sup _{\omega \in \Omega}\left|T_{0} \psi(\omega)\right| \leq M \sup _{i \in I}|\psi(i)|, \psi \in \operatorname{ran} \widetilde{A}_{0}$ ). Therefore $T_{0}$ admits a continuous extension $T:\left[\operatorname{ran} \widetilde{A}_{0}\right]^{-} \rightarrow \mathcal{C}_{b}(\Omega)$ to the closure of the range of $\widetilde{A}_{0}$ with $T \phi(\omega)=\int \phi d \kappa_{\omega}, \phi \in\left[\operatorname{ran} \widetilde{A}_{0}\right]^{-}$. By Lemma 6.2, we have $\mathcal{C}_{0}\left(\Omega_{0} / \Pi\right)=\left[\operatorname{ran} \widetilde{A}_{0}\right]^{-}$. This implies the weak continuity of $\omega \mapsto \kappa_{\omega}$.

Next we proceed to the bidualization of the partial triple $\left(E, E_{0},\{\ldots\}\right)$ $:=\mathbf{E}\left(\Omega, \Omega_{0}, m, \Pi, \kappa\right)$. As usual, we shall regard the commutative $C^{*}$-algebra $E:=\mathcal{C}_{0}(\Omega)$ with the spectral norm as a weak$^{*}$-dense subspace of the bidual $\mathbf{E}:=E^{* *} \equiv \mathcal{C}(\boldsymbol{\Omega})$ where $\Omega$ is the hyperstonian compact topological space of all norm-continuous multiplicative functionals with respect to the jointly weak*-continuous extension of the product in $E$ equipped with the weak ${ }^{*}$ topology inherited from $E^{* * *}$. That is, we identify any element $\mathbf{a} \in \mathbf{E}$ canonically with the evaluation function $\boldsymbol{\omega} \mapsto \boldsymbol{\omega}(\mathbf{a})$ on $\boldsymbol{\Omega}$.

6.7. Theorem. Let $D$ be a bounded Reinhardt domain in $E:=\mathcal{C}_{0}(\Omega)$. Then there exists a bounded Reinhardt domain $\mathbf{D}$ in $\mathbf{E}:=E^{* *} \equiv \mathcal{C}(\boldsymbol{\Omega})$ such that the canonical JB ${ }^{*}$-triple $\left(E, E_{D},\{\ldots\}_{D}\right)$ is a subtriple of $\left(\mathbf{E}, \mathbf{E}_{\mathbf{D}},\{\ldots\}_{\mathbf{D}}\right)$ and $\mathbf{E}_{\mathbf{D}}$ is the weak ${ }^{*}$ closure of $E$ in $\mathbf{E}$ and the triple product $\{\ldots\}_{\mathbf{D}}$ is the jointly weak ${ }^{*}$-continuous extension of $\{\ldots\}_{D}$.

Proof. According to Lemma 5.2, there is a positive and hence normcontinuous mapping $A: E_{D} \rightarrow F$ satisfying the identities (5.3) such that $2\{x a y\}_{D}=x A(\bar{a} y)+y A(\bar{a} x), a \in E_{D}, x, y \in E$. To study the bidual continuation of $A$, let us regard the commutative $C^{*}$-algebra $F:=\mathcal{C}_{b}(\Omega)$ as a weak $^{*}$-dense subspace of the bidual $\mathbf{F}:=F^{* *} \equiv \mathcal{C}(\widehat{\boldsymbol{\Omega}})$ where $\widehat{\boldsymbol{\Omega}}$ is a suitable compact hyperstonian topological space. Since $E=\mathcal{C}_{0}(\Omega)$ is a closed multiplicative ideal in $F$ and $E_{D}$ is a closed multiplicative ideal in $E$, also the 
weak* closures $\mathbf{E}=\bar{E}^{w^{*}}$ and $\mathbf{E}_{0}:={\overline{E_{0}}}^{w^{*}}$ are weak*-closed M-ideals in $\mathbf{F}$. Hence we may assume without loss of generality that

$$
\mathbf{E}=\{\mathbf{f} \in \mathbf{F}: \mathbf{f}(\widehat{\boldsymbol{\Omega}} \backslash \boldsymbol{\Omega})=0\}, \quad \mathbf{E}_{0}=\left\{\mathbf{f} \in \mathbf{F}: \mathbf{f}\left(\widehat{\boldsymbol{\Omega}} \backslash \boldsymbol{\Omega}_{0}\right)=0\right\}
$$

for some open-closed subsets $\boldsymbol{\Omega}_{0} \subset \boldsymbol{\Omega} \subset \widehat{\boldsymbol{\Omega}}$, and the biadjoint $A^{* *}$ maps $\mathbf{E}_{0}$ into $\mathbf{F}$. Consider the operation

$$
\left\{\text { xay }_{* *}:=\frac{1}{2}\left[A^{* *}(\mathbf{x} \overline{\mathbf{a}})\right] \mathbf{y}+\frac{1}{2}\left[A^{* *}(\mathbf{y} \overline{\mathbf{a}})\right] \mathbf{x}, \quad \mathbf{a} \in \mathbf{E}_{0}, \mathbf{x}, \mathbf{y} \in \mathbf{E} .\right.
$$

Since the biadjoint of any positive linear operator (between Banach lattices) is weak ${ }^{*}$-continuous and positive, and since the product in $\mathbf{F}$ is separately weak*-continuous, the product (6.8) is a separately weak ${ }^{*}$-continuous extension of the triple product $\{\ldots\}_{D}$. From (5.3) it also follows that $A^{* *}\left(\mathbf{f} A^{* *}(\mathbf{g})\right)$ $=A^{* *}\left(\mathbf{g} A^{* *}(\mathbf{f})\right)$ and $\mathbf{a} A^{* *}\left(\mathbf{f} A^{* *}(\mathbf{g})\right)=\mathbf{a} A^{* *}(\mathbf{f}) A^{* *}(\mathbf{g})$ for all $\mathbf{a}, \mathbf{f}, \mathbf{g} \in \mathbf{E}_{0}$. Thus $A^{* *}: \mathbf{E}_{0} \rightarrow \mathbf{F}$ is a positive linear operator with the property (5.3) and, by Lemma 5.2 , the operation $\{\ldots\}_{* *}$ is a partial Jordan ${ }^{*}$-triple product.

To complete the proof, it remains to verify axioms (J4), (J5) for the product $\{\ldots\}_{* *}$ with some bounded circular domain $\mathbf{B} \subset \mathbf{E}$. The weak ${ }^{*}$-closure of the domain $D$ seems a tempting but technically unsuitable choice for $\mathbf{B}$ in our setting. Instead we proceed as follows. Let $\boldsymbol{\Omega}_{1}:=\boldsymbol{\Omega} \backslash \boldsymbol{\Omega}_{0}$ and regard $\mathbf{E}$ as the $\ell^{\infty}$-direct sum of the weak ${ }^{*}$-closed ideals $\mathbf{E}_{0}$ and $\mathbf{E}_{1}:=\{\mathbf{f} \in \mathbf{F}$ : $\left.\mathbf{f}\left(\widehat{\boldsymbol{\Omega}} \backslash \boldsymbol{\Omega}_{1}\right)=0\right\}$. Define

where

$$
\mathbf{B}:=\mathbf{B}_{0}+\mathbf{B}_{1}
$$

$$
\mathbf{B}_{0}:=\operatorname{int}_{\mathbf{E}_{0}} \overline{D \cap E_{D}} w^{*}, \quad \mathbf{B}_{1}:=\left\{\mathbf{x} \in \mathbf{E}_{1}: \max |\mathbf{x}|<1\right\} .
$$

Recall $[5,3]$ that the bidual of a (full) $J B^{*}$-triple is a $J B^{*}$-triple with the separately weak ${ }^{*}$-continuous extension of the triple product. Hence, since the set $B_{0}:=D \cap E_{D}$ is the open unit ball of the canonical norm $\|a\|_{\{\ldots\}_{D}}:=$ $\left[\max \operatorname{Sp}\left[E_{D} \ni c \mapsto\{a a c\}_{D}\right]\right]^{1 / 2}$ on $E_{D}$, its weak*-closure $\mathbf{B}_{0}$ is the norm closure of the open unit ball of the norm $\|\cdot\|_{\{\ldots\}_{* *}}$ on $\mathbf{E}_{0}$. We show that actually $\mathbf{B}_{0}$ is a (bounded symmetric) complete Reinhardt domain in the function space $\mathbf{E}_{0} \simeq \mathcal{C}\left(\boldsymbol{\Omega}_{0}\right)$. Indeed, by Lemma 5.2 we have $A(f A(g)) \mid \Omega_{0}=$ $A(f) A(g) \mid \Omega_{0}$ for $f, g \in E_{D}$. Hence $A^{* *}\left(\mathbf{f} A^{* *}(\mathbf{g})\right)\left|\boldsymbol{\Omega}_{0}=A^{* *}(\mathbf{f}) A^{* *}(\mathbf{g})\right| \boldsymbol{\Omega}_{0}$ for $\mathbf{f}, \mathbf{g} \in \mathbf{E}_{0}$. Since $\mathbf{B}_{0}$ is the canonical unit ball of the triple product $\{\ldots\}_{* *}$ restricted to $\mathbf{E}_{0}^{3}$, Lemma 5.2 implies the Reinhardt property of $\mathbf{B}_{0}$. On the other hand, $\mathbf{B}_{1}$ is trivially a (bounded symmetric) complete Reinhardt domain in $\mathbf{E}_{1} \simeq \mathcal{C}\left(\boldsymbol{\Omega}_{1}\right)$. Since $\left(\mathbf{E}_{0}, \mathbf{E}_{0},\{\ldots\}_{* *} \mid \mathbf{E}_{0}^{3}\right)$ is a (full) $J B^{*}$-triple, for each $\mathbf{a} \in \mathbf{E}_{0}$, the operator $L(\mathbf{a}) \mathbf{x}:=\{\mathbf{a a x}\}_{* *}, \mathbf{x} \in \mathbf{E}$, is $\mathbf{B}_{0}$-hermitian. On the other hand, the positivity of $A^{* *}$ (in the sense that it preserves the cone of all non-negative functions) entails the positivity of all $L(\mathbf{a}), \mathbf{a} \in \mathbf{E}_{0}$. Hence (J4) is immediate for the partial Jordan ${ }^{*}$-triple $\left(\mathbf{E}, \mathbf{E}_{0},\{\ldots\}_{* *}\right)$ with $\mathbf{B}$ in the role of $B$ there. To establish (J5), we only have to see that given any $\mathbf{a} \in \mathbf{E}_{0}$, the operator $L(\mathbf{a})$ is $\mathbf{B}$-hermitian. We have $L(\mathbf{a})=\frac{1}{2} L_{0}(\mathbf{a})+\frac{1}{2} L_{1}(\mathbf{a})$ 
where $L_{0}(\mathbf{a}) \mathbf{x}:=A^{* *}\left(|\mathbf{a}|^{2}\right) \mathbf{x}$ and $L_{1}(\mathbf{a}) \mathbf{x}:=A^{* *}(\mathbf{x} \overline{\mathbf{a}}) \mathbf{a}$. The operator $L_{1}(\mathbf{a})$ is multiplication with a non-negative function in $\mathbf{E}$ and hence necessarily both $\mathbf{B}_{0^{-}}$and $\mathbf{B}_{1}$-hermitian. For $L_{0}(\mathbf{a})$ we have $L_{0}(\mathbf{a}) \mathbf{E} \subset \mathbf{F a} \subset \mathbf{F E}_{\mathbf{0}}=\mathbf{E}_{0}$ and $L_{0}(\mathbf{a}) \mathbf{E}_{1}=A^{* *}\left(\mathbf{E}_{1} \overline{\mathbf{a}}\right) \mathbf{a}=A^{* *}(0) \mathbf{a}=0$. Thus the complementary ideals $\mathbf{E}_{0}$ and $\mathbf{E}_{1}$ are invariant subspaces of the operator $L(\mathbf{a})$ which acts on $\mathbf{E}_{k}$ as a $\mathbf{B}_{k}$-hermitian operator for both $k=0,1$. Therefore $L(\mathbf{a})$ is $\mathbf{B}=\mathbf{B}_{0}+\mathbf{B}_{1^{-}}$ hermitian.

\section{Appendix}

7.1. TheOREM. Let $\Omega$ be a locally compact Hausdorff space and $\phi$ : $\mathcal{C}_{0}(\Omega)^{N} \rightarrow \mathbb{R}$ a continuous positive $N$-linear form (that is, $\Phi\left(f_{1}, \ldots, f_{N}\right) \geq 0$ for $\left.f_{1}, \ldots, f_{N} \geq 0\right)$. Then with $f_{1} \otimes \cdots \otimes f_{N}:\left(\omega_{1}, \ldots, \omega_{N}\right) \mapsto \prod_{k=1}^{N} f_{k}\left(\omega_{k}\right)$ we have

$$
\Phi\left(f_{1}, \ldots, f_{N}\right)=\int f_{1} \otimes \cdots \otimes f_{N} d \mu, \quad f_{1}, \ldots, f_{N} \in \mathcal{C}_{0}(\Omega),
$$

for some bounded Radon measure $\mu$ on $\Omega^{N}$.

Proof. Consider the family $\mathbf{U}$ of all finite minimal open coverings of $\Omega$ including at most one non-precompact member. That is, each $\mathcal{U} \in \mathbf{U}$ can be written in the form $\mathcal{U}=\left\{U_{1}, \ldots, U_{m}\right\}$ where $\Omega=\bigcup_{k=1}^{m} U_{k}$ with open sets $U_{k}$ such that $U_{1}, \ldots, U_{m-1}$ have compact closure in $\Omega$ and $\bigcup_{i \in I} U_{i} \neq \Omega$ whenever $I$ is a proper subset of $\{1, \ldots, m\}$. The latter property means that the $\mathcal{U}$ is minimal. This minimality property guarantees that for any covering $\mathcal{U} \in \mathbf{U}$ we can fix a system $\left\{\mathcal{U}_{\omega_{U}}: U \in \mathcal{U}\right\}$ of points such that

$$
\mathcal{U}_{\omega_{U}} \in U \backslash \bigcup_{U \neq V \in \mathcal{U}} V, \quad U \in \mathcal{U} .
$$

Since locally compact spaces are paracompact, we can also choose a partition of unity $\left\{\mathcal{U}_{\varphi_{U}}: U \in \mathcal{U}\right\}$ subordinated to the covering $\mathcal{U}$. That is, $\sum_{U \in \mathcal{U}}^{\mathcal{U}} \varphi_{U}$ $=1$ where $0 \leq \mathcal{U}_{\varphi_{U}} \in \mathcal{C}(\Omega)$ with $\mathcal{U}_{\varphi_{U}}(\Omega \backslash U)=0$. Notice that necessarily $\mathcal{u}_{\varphi_{U}}\left(\mathcal{U}_{\omega_{V}}\right)=\bar{\delta}_{U V}(=1$ if $U=V, 0$ otherwise). Hence the linear operator

$$
P_{\mathcal{U}} f:=\sum_{\substack{U \in \mathcal{U} \\ \text { precompact }}} f\left(\mathcal{U}_{\omega_{U}}\right) \mathcal{U}_{\varphi_{U}}, \quad f \in \mathcal{C}_{0}(\Omega),
$$

is a projection of $\left.\mathcal{C}_{0}(\Omega)\right)$ onto its finite-dimensional subspace with linear basis $\left\{\mathcal{U} \varphi_{U}: U \in \mathcal{U}\right.$ precompact $\}$.

The class $\mathbf{U}$ has the natural net ordering $\mathcal{U} \prec \mathcal{V}$ of being finer. That is, $\mathcal{U} \prec \mathcal{V}$ if for each $V \in \mathcal{V}$ there exists $U \in \mathcal{U}$ with $V \subset U$. It is wellknown that, given any $f \in \mathcal{C}_{0}(\Omega)$ and $\varepsilon>0$, there exists $\mathcal{U} \in \mathbf{U}$ such that $\sup _{\omega_{1}, \omega_{2} \in U}\left|f\left(\omega_{1}\right)-f\left(\omega_{2}\right)\right| \leq \varepsilon$ for all $U \in \mathcal{U}$. This means that

$$
\lim _{\mathcal{U} \in \mathbf{U}}\left\|P_{\mathcal{U}} f-f\right\|=0, \quad f \in \mathcal{C}_{0}(\Omega) .
$$


Consider the linear functionals

$$
\widehat{\Phi}_{\mathcal{U}} \widehat{f}:=\sum_{\substack{U_{1}, \ldots, U_{N} \in \mathcal{U} \\ \text { precompact }}} \widehat{f}\left(\mathcal{U}_{\omega_{U_{1}}}, \ldots, \mathcal{U}_{\omega_{U_{N}}}\right) \Phi\left(\mathcal{U}_{\varphi_{U_{1}}}, \ldots,{ }^{\mathcal{U}} \varphi_{\varphi_{U_{N}}}\right)
$$

on $\mathcal{C}_{0}\left(\Omega^{N}\right)$. Observe that, for $f_{1}, \ldots, f_{N} \in \mathcal{C}_{0}(\Omega)$,

$$
\Phi\left(P_{\mathcal{U}} f_{1}, \ldots, P_{\mathcal{U}} f_{N}\right)=\widehat{\Phi}_{\mathcal{U}}\left(f_{1} \otimes \cdots \otimes f_{N}\right) .
$$

Since the form $\Phi$ is assumed to be positive, if $-1 \leq \widehat{f} \leq 1$ we have

$$
\sum_{\substack{U_{1}, \ldots, U_{N} \in \mathcal{U} \\ \text { precompact }}}(-1) \Phi\left(\mathcal{U}_{\varphi_{U_{1}}}, \ldots, \mathcal{U}_{\varphi_{U_{N}}}\right) \leq \widehat{\Phi}_{\mathcal{U}} \widehat{f} \leq \sum_{\substack{U_{1}, \ldots, U_{N} \in \mathcal{U} \\ \text { precompact }}} \Phi\left(\mathcal{U}_{\varphi_{U_{1}}}, \ldots, \mathcal{U}_{\varphi_{U_{N}}}\right),
$$

which shows that

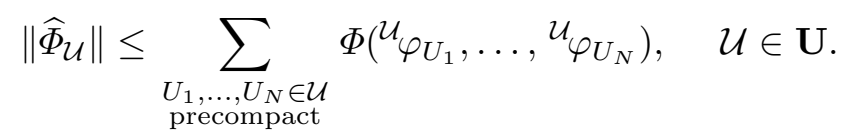

On the other hand, the functions $\mathcal{U}_{f}:=\sum_{U \in \mathcal{U} \text { precompact }} \mathcal{U}_{\varphi_{U}}$ satisfy

$$
\begin{aligned}
& 0 \leq \mathcal{U}_{f} \leq 1, \\
& 0 \leq \Phi\left(\mathcal{U}_{f}, \ldots, \mathcal{U}_{f}\right)=\sum_{\substack{U_{1}, \ldots, U_{N} \in \mathcal{U} \\
\text { precompact }}} \Phi\left({ }^{\mathcal{U}} \varphi_{U_{1}}, \ldots,{ }^{\mathcal{U}} \varphi_{U_{N}}\right), \quad \mathcal{U} \in \mathbf{U} .
\end{aligned}
$$

Hence

$$
\begin{aligned}
\left\|\widehat{\Phi}_{\mathcal{U}}\right\| & =\sum_{\substack{U_{1}, \ldots, U_{N} \in \mathcal{U} \\
\text { precompact }}} \Phi\left({ }^{\mathcal{U}} \varphi_{U_{1}}, \ldots, \mathcal{U}_{\varphi_{U_{N}}}\right) \\
& \leq\|\phi\|\left(:=\sup _{\left\|f_{1}\right\|=\cdots=\left\|f_{N}\right\|=1}\left|\Phi\left(f_{1}, \ldots, f_{N}\right)\right|\right) .
\end{aligned}
$$

By the continuity of $\Phi$ we have $\|\Phi\|<\infty$. According to the AlaogluBourbaki theorem, the bounded net $\left(\widehat{\Phi}_{\mathcal{U}}\right)_{\mathcal{U} \in \mathbf{U}}$ admits cluster points in the dual of $\mathcal{C}_{0}(\Omega)$ in the weak* sense. (Actually one could even prove its weak* convergence but we do not need this finer argument). By taking any cluster point $\widehat{\Phi}$ of $\left(\widehat{\Phi}_{\mathcal{U}}\right)_{\mathcal{U} \in \mathbf{U}}$, for all $f_{1}, \ldots, f_{N} \in \mathcal{C}_{0}(\Omega)$ we have

$$
\begin{aligned}
\Phi\left(f_{1}, \ldots, f_{N}\right) & =\lim _{\mathcal{U} \in \mathbf{U}} \Phi\left(P_{\mathcal{U}} f_{1}, \ldots, P_{\mathcal{U}} f_{N}\right)=\lim _{\mathcal{U} \in \mathbf{U}} \widehat{\Phi}_{\mathcal{U}}\left(f_{1} \otimes \cdots \otimes f_{N}\right) \\
& =\widehat{\Phi}\left(f_{1} \otimes \cdots \otimes f_{N}\right) .
\end{aligned}
$$

The proof is complete.

\section{References}

[1] T. Barton, Biholomorphic equivalence of bounded Reinhardt domains, Ann. Scuola Norm. Sup. Pisa Cl. Sci. 13 (1986), 1-13. 
[2] T. Barton, S. Dineen and R. M. Timoney, Bounded Reinhardt domains in Banach spaces, Compos. Math. 59 (1986), 265-321.

[3] T. J. Barton and R. M. Timoney, Weak ${ }^{*}$-continuity of Jordan triple products and applications, Math. Scand. 59 (1986), 177-191.

[4] R. Braun, W. Kaup and H. Upmeier, On the automorphisms of symmetric and Reinhardt domains in complex Banach spaces, Manuscripta Math. 25 (1978), 97133.

[5] S. Dineen, Complete holomorphic vector fields on the second dual of a Banach space, Math. Scand. 59 (1986), 131-142.

[6] J. M. Isidro and L. L. Stachó, Holomorphic Automorphism Groups in Banach Spaces, North-Holland Math. Stud. 105, North-Holland, Amsterdam, 1985.

[7] - - - Holomorphic invariants of continuous bounded symmetric Reinhardt domains, Acta Sci. Math. (Szeged) 71 (2004), 105-119.

[8] W. Kaup, A Riemann mapping theorem for bounded symmetric domains in complex Banach spaces, Math. Z. 138 (1983), 503-529.

[9] W. Kaup and J.-P. Vigué, Symmetry and local conjugacy on complex manifolds, Math. Ann. 286 (1990), 329-340.

[10] D.-P. Panou, Bounded bicircular domains in $\mathbf{C}^{n}$, Manuscripta Math. 68 (1990), 373-390.

[11] H. H. Schaefer, Banach Lattices of Positive Operators, Grundlehren Math. Wiss. 215, Springer, Berlin, 1974.

[12] L. L. Stachó, A projection principle concerning biholomorphic automorphisms, Acta Sci. Math. (Szeged) 44 (1982), 99-124.

[13] - On the spectrum of inner derivations in partial Jordan triples, Math. Scand. 66 (1990), 242-248.

[14] - On the classification of bounded circular domains, Proc. Roy. Irish Acad. Ser. A 91 (1991), 219-238.

[15] - On the structure of inner derivations in partial Jordan-triple algebras, Acta Sci. Math. (Szeged) 60 (1995), 619-636.

[16] —, Banach-Stone type theorem for lattice norms in $C_{0}$-spaces, ibid. 73 (2007), 193-208.

[17] L. L. Stachó and B. Zalar, Symmetric continuous Reinhardt domains, Arch. Math. (Basel) 81 (2003), 50-61.

[18] T. Sunada, On bounded Reinhardt domains, Proc. Japan Acad. 50 (1974), 119-123.

[19] J.-P. Vigué, Automorphismes analytiques des domaines produits, Ark. Mat. 36 (1998), 177-190.

[20] I. Villanueva, Integral multilinear forms on $C(K, X)$ spaces, Czechoslovak Math. J. 54 (2004), 373-378.

Bolyai Institute

Aradi Vértanúk tere 1

H-6725 Szeged, Hungary

E-mail: stacho@math.u-szeged.hu

Received January 25, 2007

Revised version December 20, 2007 